\title{
A Survey of Cybersecurity Certification for the Internet of Things
}

SARA N. MATHEU, University of Murcia, Department of Information and Communications Engineering JOSÉ L. HERNÁNDEZ-RAMOS, European Commission, Joint Research Centre, Ispra 21027, Italy ANTONIO F. SKARMETA, University of Murcia, Department of Information and Communications Engineering

GIANMARCO BALDINI, European Commission, Joint Research Centre, Ispra 21027, Italy

In recent years, cybersecurity certification is gaining momentum as the baseline to build a structured approach to mitigate cybersecurity risks in the Internet of Things (IoT). This initiative is driven by industry, governmental institutions, and research communities, which have the goal to make IoT more secure for the end-users. In this survey, we analyze the current cybersecurity certification schemes, as well as the potential challenges to make them applicable for the IoT ecosystem. We also examine current efforts related to risk assessment and testing processes, which are widely recognized as the processes to build a cybersecurity certification framework. Our work provides a multidisciplinary perspective of a possible IoT cybersecurity certification framework by integrating research and technical tools and processes with policies and governance structures, which are analyzed against a set of identified challenges. This survey is intended to give a comprehensive overview of cybersecurity certification to facilitate the definition of a framework that fits in emerging scenarios, such as the IoT paradigm.

CCS Concepts: • Security and privacy $\rightarrow$ Security requirements; Vulnerability management; $\bullet$ General and reference $\rightarrow$ Surveys and overviews;

Additional Key Words and Phrases: Security certification, IoT, security, security testing, security risk assessment, labelling

\section{ACM Reference format:}

Sara N. Matheu, José L. Hernández-Ramos, Antonio F. Skarmeta, and Gianmarco Baldini. 2020. A Survey of Cybersecurity Certification for the Internet of Things. ACM Comput. Surv. 53, 6, Article 115 (December 2020), 36 pages.

https://doi.org/10.1145/3410160

\footnotetext{
This work was supported in part by the Spanish Ministry of Economy and Competitiveness and the ERDF funds cofinantiation through the PERSEIDES project under Grant TIN2017-86885-R and in part by the European Commission through the H2020-780139 SerIoT project and the H2020-830929 CyberSec4Europe project. The research has been also supported by the the FPU-16/03305 research contract of the Ministry of Education and Professional Training of Spain.

Authors' addresses: S. N. Matheu and A. F. Skarmeta, University of Murcia, Department of Information and Communications Engineering, Murcia, 30100, Spain; emails: \{saranieves.matheu, skarmeta\}@um.es; J. L. Hernández-Ramos, European Commission, Joint Research Centre, Ispra 21027, Italy; email: jose-luis.hernandez-ramos@ec.europa.eu; G. Baldini, European Commission, Joint Research Centre, Ispra 21027, Italy; email: gianmarco.baldini@ec.europa.eu.

Permission to make digital or hard copies of all or part of this work for personal or classroom use is granted without fee provided that copies are not made or distributed for profit or commercial advantage and that copies bear this notice and the full citation on the first page. Copyrights for components of this work owned by others than ACM must be honored. Abstracting with credit is permitted. To copy otherwise, or republish, to post on servers or to redistribute to lists, requires prior specific permission and/or a fee. Request permissions from permissions@acm.org.

(C) 2020 Association for Computing Machinery.

0360-0300/2020/12-ART115 \$15.00

https://doi.org/10.1145/3410160
} 


\section{INTRODUCTION}

We live in an increasingly technology-dependent world. While pervasive and ubiquitous technologies provide significant benefits to the users, cybersecurity concerns must also be addressed, because they can undermine users' trust and hamper the development of new Information and Communication Technology (ICT) components and products. Toward this end, cybersecurity certification is one of the most powerful instruments to address cybersecurity concerns before market deployment. Among the different definitions from standardization bodies, according to the Committee on National Security Systems (CNSS) [55], cybersecurity certification can be defined as a "Comprehensive evaluation of an information system component that establishes the extent to which a particular design and implementation meets a set of specified security requirements." In Europe, the recent Cybersecurity Act [153] entered into force on 27th June, 2019. This EU new regulation gives a key role to the European Union Agency for Network and Information Security (ENISA) through a permanent mandate for the establishment and management of the EU cybersecurity certification framework. In the U.S., the National Institute of Standards and Technology (NIST) proposed a Cybersecurity Framework [145] based on standards and guidelines to help organizations manage cybersecurity risks. This framework was defined in 2014 [144] based on the Executive Order 13636, "Improving Critical Infrastructure Cybersecurity," ${ }^{11}$ and updated in 2018 to address security requirements in emerging scenarios [145]. Since then, other NIST publications, such as the recent NIST 800-37-R2 “Risk Management Framework for Information Systems and Organizations" [146], are being aligned to the framework.

Recent events like the well-known Facebook-Cambridge Analytica scandal, ${ }^{2}$ are producing an increase in the awareness of cybersecurity aspects in end-users. From the legal point of view, cybersecurity certification must deal with the laws, regulations, and directives in this area, such as the Directive on Security of Network and Information Systems (NIS Directive) [1]. Therefore, the dialogue between the manufacturers of new ICT products and policy-makers is key for the development of ICT components that comply with these regulations. From a technical perspective, currently, there are a number of different cybersecurity certification schemes. However, most of these schemes require expensive and time-consuming processes, which may be an impediment for their adoption in specific markets where the time-to-market is short or the profit margins are thin (e.g., as in the Internet of Things domain). Furthermore, they are often created for a specific scenario, domain, or geopolitical area, hindering the creation of a harmonized certification ecosystem at global level.

During the past decade, one of the most relevant scenarios for cybersecurity is represented by the Internet of Things (IoT) [8], which embraces different technologies and protocols to interconnect physical devices to the Internet infrastructure. Indeed, cybersecurity aspects have traditionally represented the most important barrier for extending the adoption of IoT systems [83, 115]. It should be noted that we consider the definition of IoT system provided by ISO/IEC 20924:2018 [95] as any system providing functionalities of IoT and inclusive of IoT devices, IoT gateways, sensors and actuators. ${ }^{3}$ For the rest of the article, we will consider that an IoT system will be composed by one or more devices. Therefore, the security level of the system will depend on the security of each device composing such IoT system.

\footnotetext{
${ }^{1}$ https://www.dhs.gov/publication/eo-13636-ppd-21-fact-sheet.

${ }^{2}$ https://www.theguardian.com/technology/2018/jul/11/facebook-fined-for-data-breaches-in-cambridge-analyticascandal.

${ }^{3}$ For a complete definition of these terms, the reader can use the ISO/IEC 20924:2018 standard [95].
} 
Well-known attacks and weaknesses, such as the Mirai Botnet [104], or the vulnerabilities on some cardiac devices reported by the Food and Drug Administration (FDA) in the U.S., ${ }^{4}$ highlight the potential impact of having vulnerable or insecure IoT systems. Indeed, with the advent of the IoT, the challenges of existing certification schemes are exacerbated due to scalability issues (many different models of IoT systems), frequent update lifecycle, limited time-to-market, and limited profit margins due to the fierce market competition. Moreover, there is a growing concern on IoT cybersecurity vulnerabilities, which must be addressed by defining a baseline security protection. A suitable certification scheme for IoT could ensure a base security level before the market deployment, where the product has been validated against specific security and privacy requirements. This is particularly useful in the IoT due to the current fragmentation of technologies and protocols, and the sensitivity level of the processed data that could be unknown when manufacturing the device.

On the basis of these considerations, this survey provides a comprehensive overview of the current cybersecurity certification schemes, which are assessed against a set of challenges identified from current institutional and research studies that are tailored to the specific features of IoT. Then, we focus on security risk assessment and testing mechanisms, which we have considered as the main building blocks for cybersecurity certification in our previous works [117, 119]. Indeed, these mechanisms are considered by the security risk assessment and testing methodology proposed by the European Telecommunications Standards Institute (ETSI) [69] as the main building blocks for security assessment. This concept is usually considered as the technical area of cybersecurity certification by evaluating the security level of a certain system against a set of requirements [55, 143].

Our Contribution: To the best of our knowledge, this is the first survey on cybersecurity certification schemes and related challenges for their potential application into the IoT. Furthermore, even if some works have analyzed different aspects related to security testing (e.g., current challenges [39] or existing testing tools [63]) and risk assessment (e.g., on modelling [106]) in IoT, we survey research proposals for both processes that are considered as key building blocks for the cybersecurity certification process. For the elaboration of this work, we applied a systematic approach by using ACM Digital Library, IEEE Xplore, and Google Scholar databases. As a first step, we identified the main cybersecurity certification schemes through existing analysis, such as References [66] and [53]. Then, for the review of security testing and risk assessment process, we use the main approaches considered by previous surveys (e.g., Reference [74]), and use them as keywords that we use in our searches (e.g., "model-based testing," "internet of things"). Considering these aspects, the main contributions of this work are:

- An analysis of the main cybersecurity certification schemes and their suitability to be considered in IoT scenarios according to a set of identified challenges.

- A systematic review of security risk assessment and testing works in IoT based on the ETSI integrated methodology for security assessment.

- A set of recommendations and research directions derived from our analysis to foster the adoption of cybersecurity certification approaches.

This survey article has the following structure: In Section 2.2, we analyze some of the most significant challenges towards the creation of a cybersecurity certification framework nowadays. Section 3 describes the main existing cybersecurity certification approaches, which are compared through the identified challenges. Thus, Section 4 presents the different schemes and associated challenges for security risk assessment. An overview of testing techniques is provided in Section 5.

\footnotetext{
${ }^{4}$ https://www.fda.gov/NewsEvents/Newsroom/FDAInBrief/ucm573853.htm.
} 
Each of these sections provides an analysis on the applicability of the identified techniques to the IoT domain. Section 6 summarizes the key findings and proposes recommendations on potential ways forward for IoT cybersecurity certification. Section 7 concludes this article.

\section{CHALLENGES OF THE CYBERSECURITY CERTIFICATION LIFECYCLE IN IOT}

This section aims to identify and describe the main challenges in cybersecurity certification. This analysis is based on a number of inputs created by various organizations such as ENISA, ${ }^{5} \mathrm{ECSO},{ }^{6}$ the Alliance for Internet of Things Innovation (AIOTI) ${ }^{7}$ and DIGITALEUROPE. ${ }^{8}$

\subsection{The Cybersecurity Certification Lifecycle}

The definition of a cybersecurity certification framework should include the processes to maintain an up-to-date security level for all the phases in the lifecycle of an IoT system. In other words, this means that the cybersecurity certification process should not stop after the initial security evaluation before the market deployment. It should represent instead a dynamic procedure during the whole IoT system's lifecycle by using complementary processes and tools. For example, this is important to mitigate zero-day vulnerabilities, which may not be known in the initial security evaluation. Here, we relate the result of the security evaluation to the certification process, which provides a cybersecurity certificate that reflects the security level achieved by the system. In this sense, the NIST [34] and the GSM Association [85] highlight that the certification process should not be stopped with the initial cybersecurity evaluation before the initial deployment, but the Ongoing life cycle must be supported.

Indeed, the security level of a certain IoT system should be upgraded after a significant update of the IoT system hardware or software, and this change should be reflected in an update of the corresponding cybersecurity certificate. It should be noted that this certificate defines the security level that is provided by a specific model of IoT system. That is, all the systems of a specific model will share the same cybersecurity certificate. The certification process must be performed by an accredited certification laboratory [67]. For example, in the EU, according to the recommendations of the ECSO WG1 [65], the certificate could be represented by a European Cyber Security Certificate (ECSC), which is intended to serve as an electronic booklet including different aspects of the certification process. First, it should provide a cybersecurity label, which summarizes in a visual way the content of the certificate obtained as a result of the cybersecurity certification process. Indeed, it would help users to compare the security levels of different ICT products. A similar concept is the EU Energy Label [70], which is widely used nowadays. This label could also include a digital representation (e.g., NFC tag or a QR code) to ease the access to the latest version of the certificate. Second, the main aspects of the evaluation, such as the name of the product, domain identifier, or validity period of the certificate, should also be contained. Furthermore, other aspects, such as list of previous certificates of a certain system or device, could help to get the traceability of the security level of that device. The ECSC should be simple enough so it can be used for end-users but, at the same time, it should give technical details that can be used by security experts.

As already mentioned, the cybersecurity certificate should evolve to faithfully reflect the security level of an IoT device. Based on this, the relationship between the cybersecurity certificate's lifecycle and the system's lifecycle is shown in Figure 1, which is composed by different stages or phases. The device's lifecycle begins with the manufacturing phase, in which the device is

\footnotetext{
${ }^{5}$ https://www.enisa.europa.eu/.

${ }^{6}$ https://www.ecs-org.eu/.

${ }^{7}$ https://aioti.eu/.

${ }^{8} \mathrm{http}: / /$ www.digitaleurope.org/.
} 


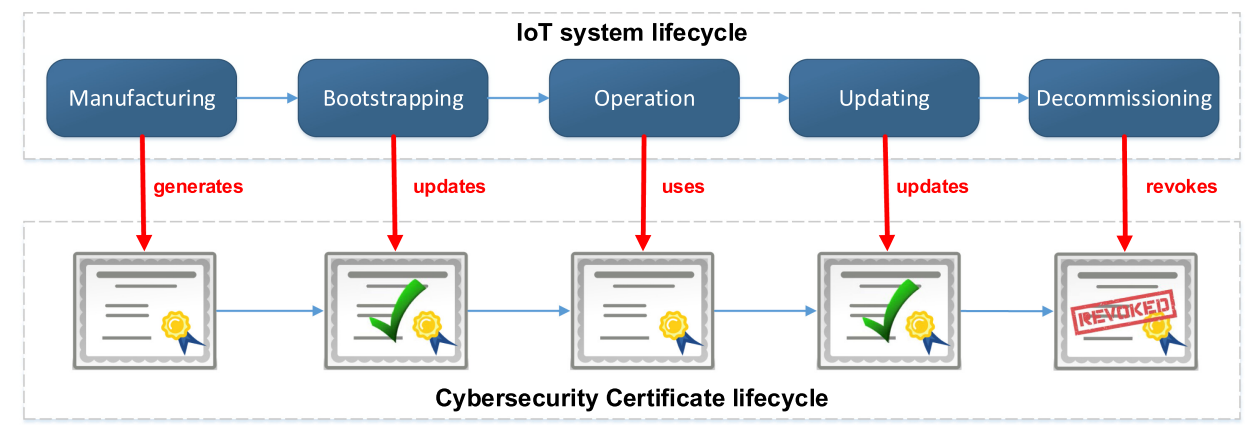

Fig. 1. The cybersecurity certificate during the IoT device lifecycle.

produced, programmed, and configured so it can be later commissioned within a network, ICT system, or infrastructure. This phase also includes a cybersecurity certification process in which the device is evaluated and certified based on security requirements. These requirements could be derived from an initial risk assessment process, and they can be mapped to a specific security level. Furthermore, a testing process is typically used to evaluate those requirements. As a result of the process, a cybersecurity certificate (and the corresponding label) can be generated [24]. The label concept improves transparency on the cybersecurity of the IoT devices, because end-users can use it to compare different devices and facilitate the understanding of the cybersecurity aspects of an IoT device. The bootstrapping phase usually includes a set of processes by which an IoT device joins a network in a specific domain (e.g., eHealth, smart home, automotive). During this phase, the device is installed and configured for a specific context and the context information should be embedded in the cybersecurity label (which should be up-to-date). Note that different domains can require different security levels; for instance, the same device could have different requirements if it is deployed to deal with health data or less sensitive information. The features of a specific domain should be identified and used as an input in the cybersecurity certification process, and they should be mapped to the certificate and the associated label. That is, the cybersecurity certificate should contain the information related to the domain for which the IoT device was certified. Then, the device starts the operation phase, in which it is providing its intended functionality. During this phase, the behavior of the IoT device should be monitored to identify potential vulnerabilities that have not been detected in the initial certification process (e.g., related to zero-day attacks). In this case, the use of the so-called intrusion detection systems could help to identify new vulnerabilities. Whatever the process to detect such vulnerabilities, this information must be shared with the manufacturer or provider of the IoT device. To address such threats, the manufacturer may decide that a new software or hardware update is needed (see updating phase), which can include also changes to the device configuration. If the software change is significant, this may mean that the IoT device must be submitted to a new cybersecurity certification (i.e., re-certification), where its security level and label could be updated. The realization of such a process in a cost-effective way may be difficult to implement, because in a complex system it is difficult to analyze the impact of the software or hardware change on the entire system. In addition, the dependencies may not only be limited to the device itself but also to the other connected devices This is especially challenging in the IoT field, where a huge amount of heterogeneous devices will be operating under changing conditions and domains.

Finally, the decommissioning phase is where the component or system is decommissioned from a certain domain. Because IoT devices could store sensitive information (e.g., cryptographic material), the process should ensure that such information is not accessible by unauthorized parties 
when the device is not active anymore. The cybersecurity certificate associated to the device should also be properly revoked.

\subsection{Challenges for the Definition and Deployment of an IoT Cybersecurity Certification Framework}

This subsection has the objective to identify and highlight the main challenges for the definition of a cybersecurity certification scheme in the IoT domain. Then, the aim of this section is not to identify the specific IoT vulnerabilities or threats (which has already been addressed in many recent surveys such as Reference [115]), but to identify the specific challenges for an IoT cybersecurity certification process. This does not exclude that some of the identified challenges may also be valid for ICT cybersecurity certification in general. This analysis is based on current institutional and research efforts.

2.2.1 Standardization. In spite of the limitations of the current approaches (which are addressed in the following sub-sections), a cybersecurity certification scheme should be based on the main concepts and operational aspects of existing standard approaches (such as Common Criteria (CC) [44]). Indeed, most of current initiatives try to use the same concepts and terms that were defined by these approaches. The use of standardized approaches will enable a common understanding about the implications and requirements of cybersecurity certification, and it will help to provide a more harmonized view of certification in different contexts, domains, or countries. Indeed, this aspect is especially relevant in the IoT domain, which is characterized by a still-fragmented landscape of standards. We believe that the alignment of current certification schemes is crucial to foster a homogeneous perspective on IoT cybersecurity.

2.2.2 Harmonization. Unlike the previous challenge, harmonization refers to the need that different cybersecurity certification schemes are able to coexist for the format and semantic of the specification and the certification results. Indeed, the wide range of existing cybersecurity certification schemes (see Reference [66] for a complete coverage) can make difficult the comparison of the output results of the difficult cybersecurity certification processes (especially at national levels). This negatively impacts the capability of users/consumers to compare the result of the certification process. In addition, manufacturers may engage in duplicated certification efforts for the same product, thus increasing the costs for deployment to market. In the energy sector, a report from ENISA [21] already highlighted the need for the harmonization of the cybersecurity certification results to support an improved clarity and transparency to manufacturers and users. To cope with these aspects, the certification meta-scheme proposed by ECSO [65] represents an initiative to embrace different certification approaches under a common umbrella. This effort is aligned with one of the main objectives of the EU Cybersecurity Act, to enable the coexistence of different certification schemes across sectors and countries. Indeed, IoT could be considered as a potential sector to define a specific certification scheme.

2.2.3 Time, Complexity, and Cost. The existing approaches for cybersecurity certification are often time-consuming and complex, requiring formal documentation and processes [99, 133]. Especially for small companies, this could imply that the manufacturer cannot afford the costs related to the cybersecurity certification process or that such process can impact the launch of a new product. Indeed, as described by Reference [183], the certification process of a certain IoT system or device could take more time and money than what is required in the manufacturing phase. This issue is even more relevant in case of update of an IoT device (hardware update or more often a software update), which may require a re-evaluation and re-certification of the IoT device (see next section on Dynamic Environment). While this is relatively common in ICT infrastructure, 
time and cost of certification are particularly relevant in the IoT domain characterized by small profit margins, limited time-to-market, and frequent software updates.

2.2.4 Dynamic Environment. IoT systems and devices will be often operating in physical environments with changing conditions that could affect their security level. Therefore, the security level of a certain system will change depending on the deployment aspects where it can be exposed to different security attacks. Indeed, at the end of the cybersecurity certification process, such system could be evaluated against a set of security requirements, but the initial tested configuration and security level can change $[16,99,133]$ due to software updates (which may also be done to address security threats), changes in the system configuration, or the need to be used in different operational contexts. Taking into consideration that software updates and patching are quite frequent in the IoT context, this requires the design of a lightweight re-certification process to reflect the changes introduced in the IoT system or device.

2.2.5 Influence of the Context. The context where the system will eventually be deployed should be taken into consideration in the cybersecurity certification process. As a main aspect of the cybersecurity certification, the context should embrace the purpose of the system, as well as the sensitivity of the data that will be managed. For example, a certain system or device could be used to handle temperature data or to collect data on the health status of a patient. This aspect is especially challenging, because it could not be known a priori when the system or device is manufactured. Indeed, an IoT system could manage different types of data throughout its lifecycle.

2.2.6 Transparency to the End-user. The transparency of the cybersecurity certification results to the end-user is a common problem, as the user may not easily perceive the positive results of the security evaluation due to the complex jargon of security certification processes. The negative consequence is that the user fails to understand the added value of the cybersecurity certification, thus decreasing the appeal of such process in the market. Different certification standards can also create confusion in end-users, because the certification results are not easily comparable. To address this challenge, a cybersecurity label (already mentioned in Section 2.1) should provide a concise representation of the security level of a certain system or device. Furthermore, it should provide a clear visibility of such security level that is obtained through the certification process [6]. Major companies, such as Bosch [90], pointed out that customers should have the capability to compare the level of security, which is provided by different systems in an easy and transparent way while the cybersecurity certification results are often technically quite complex. In fact, the label concept is intended to address an important trade-off: On the one hand, it should hide the complexity of the cybersecurity evaluation process and security requirements to the end-users; on the other hand, it should give a non-ambiguous and complete representation of the results of such process.

2.2.7 Support for the Certificate/label Lifecycle. As already mentioned in Section 2.1, the cybersecurity certification framework should manage the changes of the certificate (and label) according to the phases of an IoT system's lifecycle [16]. It should also be considered that a certified IoT system may still be subject to vulnerabilities or attacks, which were not evaluated during the certification either because of gaps in the cybersecurity certification process or because of not known threats (e.g., zero day threats). In addition, the label design should also take into consideration the dynamic aspects of the IoT domain. ECSO WG1 in Reference [65] pointed out that the cybersecurity label should represent a dynamic concept and provide the information of a new security level in a timely and non-ambiguous way. One possibility is to use Machine to Machine (M2M) technology such as NFC or a digital QR code, which can be easily regenerated. In this sense, a 
crucial aspect is the integration of monitoring techniques and tools (e.g., Reference [86]) to detect potential security attacks or threats in a timely way.

The specific features of the IoT domain where profit margins are low, software updates can be quite frequent and the time-to-market requires a very efficient cybersecurity certification process, making the application of cybersecurity certification in the IoT domain quite challenging. The next section analyzes the main existing cybersecurity certification schemes, which are compared against the previous set of challenges.

\section{STATE-OF-THE-ART OF CYBERSECURITY CERTIFICATION}

Cybersecurity certification has attracted an increasing interest in recent years to foster a trustworthy digital ecosystem. From an institutional perspective, at EU level, ENISA has been established by the Cybersecurity Act [153] as the central point for establishing and coordinating the EU efforts for the creation of a cybersecurity certification framework. Such framework is intended to serve as an umbrella term for different certification schemes in Europe to encourage the mutual recognition of evaluated items, therefore reducing the market fragmentation. This way, a cybersecurity certificate issued by a certain country could be validated and accepted by other EU Member States. In this sense, ENISA is intended to support the cooperation and collaboration among different stakeholders to quantitatively measure the security level provided by ICT products, services, and processes.

Beyond the EU, in the U.S., the NIST Cybersecurity Framework was published in 2014 [144] to promote cybersecurity activities in the organization's risk management processes. A new version was published in 2018 to strengthen the collaboration between private and public-sector efforts [145]. Moreover, in Japan, the Japan Information Technology Security Evaluation and Certification Scheme (JISEC) [93] was created in 2001 and promoted by the Information-technology Promotion Agency (IPA) to evaluate the security functionality of IT products. JISEC follows the Common Criteria (CC) certification scheme, which is described in Section 3.1. In addition, there are approaches that, while not focused on cybersecurity certification aspects, could help in the definition of an IoT certification approach. An example is the IoT Security Compliance Framework [94], which formulates best practices for a compliance testing process. This framework also takes into account the management of systems and data lifecycle. Another example is the IoT Trust Framework [150], which describes strategic principles to support the security of IoT devices and the associated data during their lifecycle.

Based on these considerations, this section surveys the main existing certification schemes that are widely considered for the certification of generic IT products. Furthermore, we assess the suitability of these schemes to be considered in the IoT ecosystem by using the challenges defined in the previous section. In our analysis, we noted that there is only one cybersecurity certification scheme focused on IoT that is proposed by ICSA Labs [92]. In addition, it should be noted that a summary of this analysis is provided in Table 1, which can be found in the Appendix.

\subsection{Common Criteria}

The most widely deployed and adopted cybersecurity certification standard is the Common Criteria (CC) [44]. In this standard, a certain system or product (Target of Evaluation (TOE) in CC notation) is evaluated against a set of security requirements based on a certain Protection Profile (PP). These requirements are defined in an implementation-dependent statement that is called Security Target (ST). CC supports the comparability among the results of independent cybersecurity evaluations through Collaborative Protection Profiles (cPP), under the terms of the Common Criteria Recognition Arrangement (CCRA) [56], which is aimed to cope with harmonization and standardization challenges. CC uses the Evaluation Assurance Levels (EAL), which defines specific 
assurance requirements that must be fulfilled to achieve a certain certification level. CC assurance is achieved by analyzing the processes and guidance documents, the TOE design, as well as through functional and penetration testing [195]. The concept of context is included in the PP by considering the TOE physical environment, security requirements, and the TOE purpose, which is related to the product type and the intended use.

Even if CC is the most deployed cybersecurity certification standard, various limitations have been identified by the industry and research community $[99,103]$. These limitations are related to the challenges described in the previous section: (a) time and effort required for the execution of the cybersecurity certification process, (b) the analysis of the evaluation-related documentation, which can be complex for high EALs, and (c) the management of changes in the certified product (akin to the support for the IoT system lifecycle described above). These challenges can hamper the commercialization of the IoT system or product, as they may impact time-to-market and profit margins. It is worth noting that the outcome of CC cybersecurity certification certifies a particular version of the product in a specific configuration. Therefore, any configuration or update in a certain product could require to repeat the certification process. This is especially problematic in the IoT domain, because products will be frequently evolved and updated, while the security certification must be performed on a specific version of the product. To address this problem, the designers of the CC scheme proposed the Assurance Continuity (AC) [43] re-evaluation process in the context of the Common Criteria Recognition Arrangement (CCRA). This process has the objective to define a set of minimum requirements to conduct Assurance Continuity activities that must be mutually recognized. The CC Assurance Continuity (CCAC) recognizes that not all the changes made to a certified TOE or its environment would require the repetition of the cybersecurity certification process performed previously. Therefore, the CCAC process defines an approach to minimize the redundancies in the cybersecurity evaluation. The TOE manufacturer must produce an Impact Analysis Report (IAR) that analyzes and describes the impact of changes to the already certified TOE. The IAR is submitted to an evaluation authority, which must decide from the IAR description if the proposed change is within the boundaries of the maintenance scheme or if it is a major impact, which would require re-certification.

An additional problem in the early version of CC is the lack of comparability with other certification approaches, due to a cumbersome technical documentation, which makes an objective comparison more difficult [88], and therefore, the harmonization of the cybersecurity certification outcomes. CC designers tried to address this issue through the CCRA and the cPP. Finally, CC considers the use of a label in which only the EAL is included, so the information provided is limited.

\subsection{Commercial Product Assurance}

The Commercial Product Assurance (CPA) [46] is the UK national scheme from the Communications-Electronics Security Group (CESG) for the evaluation of systems and products against CESG standards. The methodology is supported by testing approaches (mostly through black testing [134]) for the certification of a certain system to be used in the UK public sector. CPA allows the update of products' certificates throughout their lifetime if vulnerabilities are identified, and patches must be applied to address such vulnerabilities [46]. This does not mean that the system is monitored, since the re-certification will only be performed if a change on the security level is detected. In addition, although CPA is intended to provide a lightweight re-certification process, it still takes about six months to execute it [136]. Products are tested against published CPA Security Characteristics, which are published in Reference [46]. However, it should be noted that some of the proposed metrics are qualitative and they may be ambiguous. Moreover, a Mutual Recognition Agreement (MRA) has not been defined for this scheme. As a consequence, products 
certified in the UK will not be accepted in other countries, so it does not address the harmonization challenge. To deal with this issue, a mapping between CPA security statements and PPs of CC was proposed [137]. The cost of the certificate is 1,300.00 USD per day of work, and it can take between 6 and 18 months [23]. The concept of labeling is not mentioned, and the context in which the system will be used is not considered to be part of the certificate, as shown in Reference [135].

\subsection{Cybersecurity Assurance Program}

The Cybersecurity Assurance Program (CAP) is a certification methodology created by the Underwriters Laboratories (UL) company for the evaluation of products or systems by using the UL standards. In particular, CAP is based on the UL 2900 standards [177], which provides testable criteria for evaluating potential vulnerabilities in a certain product or system. However, these are proprietary standards from a for-profit organization that are not validated by experts and the research community; therefore, the scheme is not widely recognized as an accepted certification scheme. Furthermore, UL 2900 standards are not public, so harmonization and standardization aspects are not addressed. The certification process can take several months, and the result is a certificate that is valid for 12 months. To cope with the challenge related to a dynamic environment, during the certificate lifecycle, any software change must be documented and notified to evaluate the product before an updated certificate is issued. The manufacturer is also required to securely patch the TOE in a timely manner [154]. As described in Reference [178], the testing process includes fuzzy testing, code and binary analysis, structured penetration testing, risk control analysis, and security risk controls assessment. More details about these techniques are described in Section 5. Finally, the context is partially analyzed (e.g., a section of the scheme is related with health care aspects), and labeling is not considered.

\subsection{Certification de Sécurité de Premier Niveau}

The Certification de Sécurité de Premier Niveau (CSPN) [17] is a certification methodology proposed by the National Cybersecurity Agency of France (ANSSI) in 2008. According to Reference [58], "The main three objectives of CSPN are the verification of the product's compliance with its security specifications, the theoretical rate of the mechanisms and the list of the known vulnerabilities of products of the same type; and the test of the product trying to bypass its security functionality." In CSPN, the security specifications of a product or system are analyzed and tested through intrusion mechanisms. An additional aspect is that CSPN has been designed to perform evaluations in a short period of time to support the product development lifecycle.

CSPN can be considered as a more lightweight certification methodology than CC, in which a certain product or system is evaluated within a short period of time (a typical certification can take between 35 days and 2 months) with a reduced cost (about 25.000-35.000 Euros [23]). However, it is standard in France, there is not an MRA related to it, so it does not foster a harmonized cybersecurity certification approach. Moreover, the concept of labeling is not considered, and it requires a complete certification process in case of any change on the security level of a certain product. Furthermore, monitoring aspects are not contemplated to deal with the certificate lifecycle. Finally, the context is analyzed and specified in the report as a result of the certification [18], since the TOE is evaluated in a representative platform in which the product will be used.

\subsection{ICSA Labs loT Security Testing Framework}

The IoT Security Testing Framework [92] was developed by the ICSA Labs company to specify security testing requirements for different types of IoT systems. An IoT system that passes all the test cases (based on recurring testing [91]) provides assurance that it can be deployed without degrading the security or the functionality of the network in which it will be installed. In this case, 
the system and its components are awarded with the ICSA Labs IoT Certification. The approach is based on a periodic assessment and update of the certification criteria. To address evolving threats, it includes frequent iterative updates, addressing the dynamic environment challenge inherent to IoT. To manage the lifecycle of the system or device, ICSA Labs obtains a contractual commitment from the manufacturer of such systems, so they agree that the certification of the product will be updated by the corresponding standards from the company. To strengthen the assessment process, ICSA Labs (or associated accreditation bodies) also carry our random assessments of the current product. Toward this end, the corresponding entity has a short time (e.g., two to four weeks) to fix the identified issues if a product fails the evaluation. Indeed, if the product does not validate the current certification criteria by the end of this period, the ICSA Labs Certification is revoked for that product. Finally, the certificate is renewed annually by repeating the whole process. However, this framework is not focused on certification aspects, and consequently, the labeling process is not considered. Furthermore, there is no MRA for this approach, and the criteria and processes are not standardized. In addition, currently there are only three IoT products certified with this approach, due to its recent creation. For these products, the process took 1-2 months, but the cost is unknown [102].

\subsection{Other Certification Schemes}

In addition to the main certification schemes previously described, here, we discuss other approaches that are considered in specific countries or sectors. For example, more focused on privacy certification aspects, the European Privacy Seal ${ }^{9}$ is intended to certify that an IT product or service is compliant with the General Data Protection Regulation (GDPR) [71]. The European Privacy Seal can also take into consideration specific legislative instruments from the EU Member States. For the certification process, the corresponding regulatory requirements are translated into questions to be answered or addressed. The process is done manually by experts answering the related questions.

Another scheme is the Singaporean National IT Evaluation Scheme (NITES), ${ }^{10}$ which was defined by the Security Accreditation Committee (SAC) in Singapore. It is mandatory for manufacturers, which supply IT products to governmental agencies in Singapore. Although the NITES scheme specifications were not made public, this scheme represents a customization of CC standard v3.1 [66], at the approximate level of EAL4+ with additions, which are mostly targeted to the vulnerability analysis. UL is the first international test laboratory accredited by the Singapore Government to operate under the NITES.

Linked to the standards, the Software Improvement Group (SIG) Software Quality Model for Security [192] is based on ISO 25010 and reflects the extent to which security best practices are implemented. As specified in Reference [192], the SIG evalution is based on a combination of code review by experts with the use of commercial and open source tools. It describes five quality characteristics of software security that are grouped in three sets (Confidentiality and Integrity, Non-repudiation and Accountability, and Authenticity) and their relationship with different system sub-properties (best practices) from ISO 25010. The assessment is based on scores with sub-properties of weak, normal, or strong, which are linked to a mark between one and five stars for each system property that represents the quality of the implementation.

In Germany, the ULD Datenschutz-Gütesiegel ${ }^{11}$ aims to certify IT products, including software, hardware, and services, which should be used by German public authorities. The product

\footnotetext{
${ }^{9}$ https://www.european-privacy-seal.eu.

${ }^{10}$ https://www.csa.gov.sg/.

${ }^{11} \mathrm{https}: / / \mathrm{www} \cdot$ datenschutzzentrum.de/guetesiegel/.
} 
compatibility to the data protection and data security rules are established by a formal procedure where penetration testing [30] is used. A simplified certification process can be used for minor changes, while for major changes the overall assessment process must be repeated.

Finally, CTIA-The Wireless Association (CTIA), which represents the U.S. wireless communications industry, has recently presented an IoT certification scheme for IoT systems that use LTE and/or Wi-Fi technologies [61]. The certification process is based on several security characteristics grouped on different categories, such as authentication, encryption, patch management, and threat monitoring. Each aspect is assessed though the execution of tests, which are defined by the CTIA, and realized through a specific testing methodology by an accredited testing laboratory. However, it is still too early to evaluate this scheme, since currently there are no systems or devices certified with it.

From the previous analysis, there is not a silver bullet certification scheme that copes with the requirements associated to the IoT paradigm. IoT cybersecurity certification sets out unique challenges that must be addressed through the current schemes by adapting the certification process accordingly. On the one hand, IoT is currently realized through a fragmented landscape of technologies and protocols that are not standardized in some cases. Consequently, the certification process must deal with heterogeneous systems and devices. On the other hand, the scale of IoT requires lightweight and flexible approaches that are able to provide an effective and efficient certification approach throughout the lifecycle of such components. Furthermore, the involvement of end-users is crucial, since IoT systems could handle sensitive data, and they will be often managed by non-expert users. Based on the discussed schemes, CC represents the most recognized approach across the world by providing different assurance levels and a comprehensive evaluation process. However, beyond the definition of new schemes (which could hinder harmonization aspects), we claim that the cost and complexity associated to $\mathrm{CC}$ requires a revision and adaptation of the scheme to be further adopted in the IoT ecosystem.

\subsection{Driving Cybersecurity Certification through Security Risk Assessment and Testing}

As already mentioned, the scope of this survey is established around security risk assessment and testing processes as the main building blocks for security assessment or evaluation, which embraces the technical processes of security certification. Indeed, NIST [142] and CNSS [55] employ the terms of assessment and evaluation to define cybersecurity certification. With this aspect in mind, we consider the security risk assessment and testing framework provided by ETSI [69], which explicitly states the integration of security risk assessment and testing process to assess the security of an ICT system based on ISO 29119 and ISO 31000 standards [169]. Figure 2 shows the building blocks of the methodology. In particular, security risk assessment includes risk identification to detect and describe a certain risk; risk estimation aimed to define the level of the risk; and risk evaluation, which is the process to analyze if the risk is acceptable. In the case of security testing, it involves the process of test design and implementation, so test cases are designed and implemented; test environment set up and maintenance, which describes, establishes, and maintains the environment where the tests are executed; and test execution, analysis, and summary, which deals with the analysis of the results derived from test execution. As part of the Security Assessment process, the Treatment activity involves security strategies and countermeasures to cope with a certain risk. It should be noted that additional processes are considered by the ETSI methodology, including Establishing the context, which is intended to identify security objectives and the context of the assessment process. Furthermore, the activities Communication \& Consult and Monitoring and review are meant to control and react according to the results derived from the assessment process composing the management perspective of the framework. However, the analysis of these processes is out of the scope of this work. 


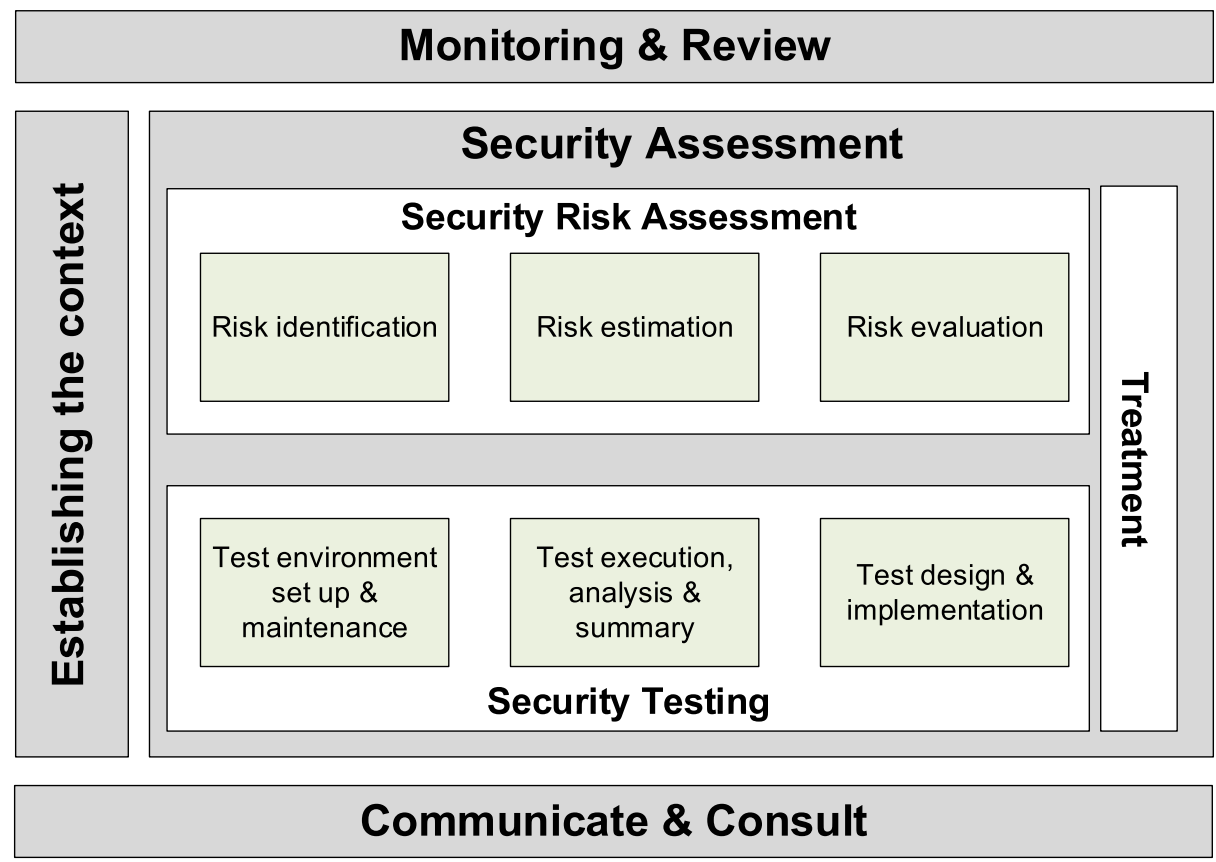

Fig. 2. Overview of the ETSI risk-based security assessment and testing framework [69].

In addition, it should be noted that other standardization organizations (e.g., NIST 800-37 [97]) and associations (e.g., ECSO [65] and Cyberwatching [116] in Europe) consider explicitly both processes as part of the certification process. Based on these aspects, the following sections analyze the main security risk assessment and testing approaches, including research proposals, and their suitability to be considered in IoT scenarios. It should be noted that other surveys exist in the scope of the integration of risk-based security assessment and testing [68] or focused on riskbased testing aspects $[76,84]$. However, such works are not focused on IoT scenarios, and they do not describe or analyze current mechanisms to cope with the challenges derived from risk-based security assessment and testing processes.

\section{SECURITY RISK ASSESSMENT: CURRENT EFFORTS}

Risk assessment is defined in CNSSI-4009 [55] as "the process of identifying, prioritizing, and estimating risks. This includes determining the extent to which adverse circumstances or events could impact an enterprise. Uses the results of threat and vulnerability assessments to identify risk to organizational operations and evaluates those risks in terms of likelihood of occurrence and impacts if they occur." As in the formal definition, we consider a risk assessment methodology to determine the risk of a vulnerability, weakness, or threat as a way to measure the security level of a certain device, component, or system based on the ETSI methodology previously described.

\subsection{Challenges on Risk Assessment in loT}

Related to the challenges described in Section 2, we describe below some of the main challenges related to the risk assessment process in the IoT domain.

4.1.1 Risk Assessment Standardization. Like in the case of the certification process, there is a plethora of risk assessment mechanisms taking into account different metrics and procedures. 
Consequently, this heterogeneous landscape of solutions makes the comparison of different devices and systems more complex. In addition to the ETSI methodology previously described, risk assessment is widely recognized as a core component of the security assessment of ICT products [97]. However, as defined in the next subsections, the current heterogeneity of risk assessment approaches hinders the adoption of current schemes in the IoT paradigm. Indeed, beyond the different approaches for risk assessment, we noticed a lack of common consensus and standards to refer the concepts related to the risk assessment process itself [156]. In this direction, we believe that the adoption of a standard-based common framework for security risk assessment is crucial to foster a homogeneous perspective on cybersecurity risks in IoT.

4.1.2 Complexity and Dynamic Environment. Common risk assessment methodologies require the production of a complex and cumbersome documentation, which may deter the application of such methodologies in the market. In the IoT context, this is especially relevant due to the high dynamism associated to IoT scenarios and the short time-to-market. While current schemes consider the use of periodic assessments to maintain the security level up-to-date, these are insufficient to deal with dynamic nature of IoT. Indeed, as described by Reference [147], the variability of IoT systems makes typical periodic assessment approaches inadequate for IoT. For that reason, there is a need to design mechanisms to anticipate new situations that could provoke new cybersecurity risks in a certain context.

4.1.3 Aggregated Security Risk Assessment. An IoT system will be usually composed by different devices that, in turn, could be composed of several components (i.e., sensors or actuators) with a different security level. It means that the risk assessment of a certain system will depend of the security level associated to each part of the system. Here the main challenge is to define a method in which each risk value could be aggregated to provide a reliable value for the whole system [27]. While most of the risk assessment schemes already consider the use of weights associated to different metrics to assess a certain product, these aggregation aspects are usually ignored.

4.1.4 Influence of the Context. Like in the overall certification process (see Section 2.2.5), the context in which the system must operate has to be taken into consideration. However, in the IoT paradigm, the context of a specific system could change during its lifecycle, so re-assessment aspects must be considered. Indeed, a certain system could be used to manage different kinds of information with different protection requirements. Therefore, assessment approaches must consider the context where the system will operate in such a way that some metrics could be more representative (i.e., with an increased weight) depending on it. For example, the risk associated to a certain system exchanging health data from a patient could have more restrictions on confidentiality than another system sending data on the level of humidity in the environment.

4.1.5 Use of Consistent Security Metrics. As already mentioned, common risk assessment approaches are based on the use of different security metrics, which are employed to provide a more reliable assessment of a product's security level. However, some of these metrics, such as likelihood or impact, are difficult to be measured (see Reference [88] for further details). As a consequence, the overall process adds a certain degree of subjectivity [164]. Indeed, this has motivated the authors of certain schemes (e.g., the Common Weakness Scoring System (CWSS) [128]) to ignore some of these metrics. These aspects are exacerbated in the IoT domain due to the dynamic and changing nature in the context where the system will be operating, as previously described.

4.1.6 Multi-layer Security Risk Assessment. Related to the aggregation of different security risk scores, the assessment process should consider the communication and security protocols that are used at different layers of the protocol stack. In this direction, the EU RASEN project [157] 
suggested an approach for a compositional risk assessment process by using the scores of different network layers. In the IoT context, such aggregation techniques must cope with the fragmented landscape of protocols that are used at different network layers, including power-efficient wireless communications technologies, such as LoRa or Sigfox [122].

Based on these challenges, below, we provide an overview of the main current risk assessment methodologies, which are compared according to the previous challenges. A summary of this analysis can be found in Table 2 in the Appendix.

\subsection{CWSS}

The Common Weakness Scoring System (CWSS) [128] represents a methodology to prioritize software weaknesses. The main motivation is to provide means to different stakeholders (e.g., software testers or manufacturers) for quantifying the risk associated to a specific weakness. This way, the corresponding stakeholder can prioritize the weakness to be solved based on the estimated risk. CWSS aims to standardize the approach to characterize weaknesses, so vendors, developers, and users can have a harmonized representation of the most critical weaknesses associated to a specific software. The approach defines the Base Finding, Attack Surface, and Environmental metric groups, which in turn contain different metrics to quantify the CWSS score associated to a certain weakness. In particular, Base Finding aims to represent the risk of the weakness, including the technical impact, internal control effectiveness, and finding confidences. The Attack Surface considers the attacker's perspective, including metrics associated to the required privilege (which they must have to access the weakness) or the deployment scope. Then, the Environmental metric group captures contextual aspects by considering weaknesses' features in a certain environment (e.g., likelihood of discovery). It should be noted that, in spite of exhaustively defining these metrics, some of them are difficult to be quantified, as highlighted by Reference [147]. Based on the scores obtained for each metric group, the final CWSS score is represented by a number between 0 and 100. Furthermore, CWSS provides a flexible methodology that can be refined by users. Indeed, it is often used with the Common Weakness Risk Analysis Framework (CWRAF) [127] to identify weaknesses in specific business domains.

\subsection{CVSS}

As a complementary approach to CWSS, the Common Vulnerability Scoring System (CVSS) [77] is another approach to rank vulnerabilities instead of software weaknesses. On the one hand, it is a similar methodology, which is also based on three group metrics: Base, Temporal, and Environmental. In this case, the Temporal metric group is intended to represent the variations of a certain vulnerability over time. On the other hand, CVSS provides a more simple approach by considering 14 metrics, which could receive a specific value from a predefined set or list. Furthermore, unlike CWSS, CVSS is focused on discovered vulnerabilities; therefore, CWSS can be used in a preliminary phase to quantify the risk associated to a weakness, which could lead to a certain vulnerability that can be assessed by CVSS. Although CVSS is similar to CWSS, some metrics like likelihood have been removed to reduce the subjectivity associated to this metric. The CVSS methodology has evolved during past years until the latest version (CVSSv3.0 [77]). This approach is widely adopted nowadays; for example, it is used in the Common Vulnerabilities and Exposures $(\mathrm{CVE})^{12}$ and in the National Vulnerability Database (NVD) ${ }^{13}$ created by the NIST.

\footnotetext{
${ }^{12}$ https://cve.mitre.org/.

${ }^{13}$ https://nvd.nist.gov/.
} 


\subsection{OCTAVE}

A different risk assessment approach is represented by the Operationally Critical Threat, Asset, and Vulnerability Evaluation (OCTAVE) [42], which was proposed by the Carnegie Mellon University's Software Engineering Institute (SEI) in collaboration with the Computer Emergency Response Team (CERT) in the U.S. The methodology covers different aspects for risk management, including the identification of relevant assets for a certain organization and the vulnerabilities and threats associated to such assets to define a strategy accordingly. It should be noted that OCTAVE focuses on operational risk and security practices, not on technological aspects related to the risk to be assessed.

The OCTAVE framework defines eight processes. However, before performing such processes an exploratory phase or Phase Zero is used to come up with the criteria to be used during the application of the methodology. This includes the evaluation of the impact in a specific area (health, productivity, financial, etc.), so contextual aspects are also considered. However, this approach does not effectively provide a detailed quantitative analysis. Furthermore, while it defines a set of metrics, the mapping with impact intervals (low, medium, and high) is open to subjective interpretation [42], so the comparison between different OCTAVE-evaluated components is more difficult. In addition, the methodology is based on a complex documentation, which has motivated the development of a more lightweight alternative called OCTAVE-S [9].

\subsection{DREAD}

The DREAD scheme [125] is used to compute a risk value associated to a certain threat or vulnerability based on the use of five categories: Damage potential, Reproducibility, Exploitability, Affected users, and Discoverability. It was used at Microsoft and currently it is used by OpenStack [151]. The context can be considered when assigning a value to each category.

The methodology produces a number between 0 and 10 that represents the associated risk. However, according to Reference [79], it is not clear how the scale should be considered; indeed, it depends on the users responsible for performing the modelling of threats. DREAD requires scoring each of the five categories on a scale from 0 to 10 , which leads to discussions on the fine differences between consecutive numbers, e.g., five and six. This problem is exacerbated when the assessment is performed by different users that must agree on these aspects. A potential solution to this issue, as remarked in Reference [139], is the use of scores of High, Medium, or Low, which are easy to agree, instead of using the 11-valued scale by Microsoft.

\subsection{OWASP Risk Rating Methodology}

The Open Web Application Security (OWASP) Risk Rating Methodology [152] is part of the OWASP project, which provides an approach to test security aspects in web applications. The risk rating methodology estimates the risk in terms of likelihood and impact by following several steps. The first one consists on identifying a risk to be rated, analyzing and gathering information about it. The second step analyzes factors for estimating the likelihood. There are different factors that can help to determine the likelihood, such as the ease of discovery and exploit, or the skills of the attacker. The third step is about identifying factors for estimating the impact.

Then, the fourth step determines the risk severity. The likelihood and impact estimations can be used to calculate an overall severity for this risk: none, low, medium, high, or critical.

The limitation of OWASP is that it is only focused on web applications. As in the other schemes, the scale (low, medium, and high) is only based on the consensus of the users, so a certain degree of subjectivity is associated to this process. 


\subsection{CERT/SEI and FMECA}

CERT/SEI [162] defines an approach to scoring the Secure Coding Standards of the programming languages C [161], C++ [25], Perl [163], Android [45], and Java [113] using the FMECA methodology [187]. CERT/SEI provides a set of guidelines and rules to identify potential failures of a product or component, and to assess the risk associated to such failures. Each guideline is intended to provide a risk assessment description regarding the potential consequences derived from ignoring a particular rule or recommendation. This information is used to prioritize the risks and to develop associated actions to cope with the most serious failures.

The approach characterizes items in terms of Severity, Likelihood, and Remediation Cost, each of them with three degrees (low, medium, and high). The three values are then multiplied for each rule. The score ranges between 1 and 27 [138].

However, CERT/SEI focuses on a specific domain (code in a certain programming language), and it can only be applicable in the development stage of a system. In addition, as in the other schemes, the assignation of the scale is subjective, and it depends on the user that assigns the risk. In addition, aggregation of different components and multilayer aspects are not considered, since it is only focused on the code review. It is worth noting that CERT/SEI is not intended to measure a risk, but prioritize good practices, so it adopts a different vision compared to other schemes for risk assessment.

\subsection{Veracode Rating System}

The Veracode Rating System [180] is based on the combination of dynamic and static analysis to evaluate weaknesses/vulnerabilities in web applications. On the one hand, static analysis is used to generate an intermediate representation (from the executable), so potential security vulnerabilities can be discovered by using scanning tools. On the other hand, dynamic analysis considers the use of penetration testing mechanisms to identity potential security threats or vulnerabilities at runtime. Then, the results are quantified by using the Security Quality Score, which represents a scoring approach based on the CVSS methodology. This system is based on the security properties of the so-called CIA triad (Confidentiality, Integrity, and Availability), so the system quantifies a level to represent the potential impact of a security breach in a certain security property. The final score is given by a number $(0-100)$ to represent a completely insecure system (0), or an application without any threat or vulnerability (100). It should be noted that this score is then weighted according to security experts, so a certain degree of subjectivity is included in the process.

\subsection{Risk Assessment: Analysis and Applicability to the loT Context}

In this section, we provide an analysis on how the risk assessment techniques described in the previous section can be applied to the IoT context. A summary of this analysis is provided in Table 3 in the Appendix.

For a suitable risk assessment approach for the IoT, it is essential to identify and analyze the threats, weaknesses, and vulnerabilities to quantify their associated risk. Related to these aspects, Reference [19] analyzes potential approaches for threat mitigation in IoT based on autonomic schemes. Furthermore, Reference [179] carries out an empirical analysis to identify potential weaknesses in real devices in the scope of power grids. Towards this end, authors considered the existing data from the NVD and CWE. Moreover, Reference [3] proposed a taxonomy to classify threats and attacks, as well as the associated attackers in IoT scenarios. In addition, References [130] and [31] are intended to give some guidelines to improve security aspects during the development lifecycle of an IoT system. However, the risk assessment proposals in the IoT domain are limited. 
Regarding the application of CVSSS/CWSS schemes, Reference [155] adapts the risk assessment formula of CVSSv2 for Bluetooth technology. In particular, they extend the authentication metric to include new security factors inherent to the Bluetooth technology. Furthermore, Reference [87] also aims to adapt the CVSS scheme to IoT by removing some metrics such as report reliability and adding other aspects, such as disclosure level. In Reference [185], CVSS is used in combination with attack graphs and maximum flows to assess the risk in an industrial IoT domain. CVSS is also used in Reference [188] to calculate the likelihood of the Fault Tree Analysis in smart homes. A more sophisticated approach is provided by Reference [191], which integrates a situation reasoning method based on semantic ontology and user-defined rules to represent vulnerabilities and attacks with the use of CVSS. Also based on these schemes, we have proposed the use of the CWSS for IoT in Reference [118] as a core component to build an automated risk assessment methodology. We proposed a similar approach in Reference [119] related to the use of CVSS that includes a practical example by using IoT protocols.

Moreover, Reference [159] proposes an adaptation of the DREAD risk model to IoT. For this, authors classify the different threats by using a threat modeling tool based on the STRIDE schema [124], a complementary approach to DREAD for threat classification. Then, they aggregate the different risk values by using different weights, which are estimated based on the type of system being evaluated. In Reference [54], DREAD is also used in the smart metering scenario. They analyze the risks of the use case that are classified through the STRIDE schema. In Reference [41], DREAD is applied to the mHealth domain due to its simplicity. In combination with STRIDE, it is used to assess the risk and to provide mitigations and countermeasures. A similar approach is proposed by Reference [149], which describes a system aimed to represent the vulnerabilities and attacks identified through STRIDE and ranked with DREAD. Another work that uses an existing approach is Reference [10], which applies OCTAVE to the smart-home domain by identifying risks and providing countermeasures specific for this domain.

Other works propose to combine several risk assessment schemes. This is the case of Reference [79], which is focused on the development of a framework for the definition of requirements, which can be used to comply to the aircraft security constraints. The framework uses CVSS, STRIDE, and DREAD schemes. Furthermore, Reference [98] makes use of the Microsoft SDL tool and STRIDE to model threats and to adapt the formulas and metrics from CVSS, OWASP, HEAVENS [11], and EVITA [72] to assess the risk in vehicular systems. Finally, Reference [96] is based on an existing approach called Information Security Risk Analysis (ISRA) that is applied to the smart-home domain with the purpose of finding potential vulnerabilities and threats. They recommend the definition of a general model for security and privacy risk, which can be included in the design of smart homes.

Beyond previous approaches that are based on existing risk assessment schemes, other works propose the development of new risk assessment methodologies that can be potentially considered for IoT. In this sense, Reference [2] describes a risk-based adaptive security framework for IoT-enabled eHealth that is intended to predict and react to attacks based on the information provided by a monitoring process. This proposal relies on context-awareness techniques and game theory. In Reference [189], authors use Bayesian networks to compute the risk probability based on different parameters to analyze the weight condition of each propagation path. Furthermore, Reference [80] uses similar metrics to CVSS in combination with a Hierarchical Attack Representation Model (HARM), based on attack paths, to assess the IoT security. Moreover, Reference [109] is based on game theory to build a vulnerability quantification method by using an attack tree for a social-IoT-based network environment, which is compared with other risk assessment methods, such as CVSS. In Reference [166], authors design a new general purpose risk assessment methodology for IoT, addressing in a systematic way attack propagation by using bipartite graphs. 
In addition, Reference [13] proposes a taxonomy of risks in the health domain to develop a risk assessment method to calculate likelihood and severity. The same authors in Reference [14] propose a general method for risk assessment by using an analytic hierarchy process based on different metrics. Furthermore, Reference [167] develops a general-purpose risk assessment scheme for IoT based on threat modeling through attack trees and dependencies graphs to obtain a risk value. Moreover, Reference [112] designs a dynamic risk assessment for IoT inspired on the Artificial Immune System. In this case, network packets are captured and analyzed looking for attacks, so risk estimation is performed based on the attack detection result. Additionally, Reference [82] proposes a risk assessment method for evaluating threats based on graph models that is applied to a building power management system.

As previously described, there is a plethora of works related to the potential application of risk assessment methods in the IoT paradigm. However, most of these proposals are focused on a specific context or they do not address some of the main challenges associated to risk assessment methodologies. In particular, current approaches fail when dealing with IoT specific aspects related to the dynamism and scale of common scenarios. Furthermore, most of these schemes add a certain degree of subjectivity to estimate the risk associated to a certain vulnerability or threat; as a consequence, the comparison of different devices or components could be pointless.

We consider that a risk assessment approach for IoT must be supported by rigorous automated and technical procedures based on testing approaches to come up with a more reliable and objective assessment. However, as previously described, current schemes are not able to cope with these challenges. Following current recommendations from other researchers [147], we claim that there is a real need to develop a new risk assessment methodology for IoT to increase end-users' trust for the development of new IoT products.

\section{SECURITY TESTING}

According to CNSSI-4009 [55], "Security Testing is the process to determine that an information system protects data and maintains functionality as intended." When a security testing technique is applied, the information system to be tested is usually called System Under Test (SUT).

\subsection{Challenges on Security Testing in loT}

Based on the challenges previously described in Section 2 for certification, the application of security testing in IoT scenarios should cope with at least the following aspects.

5.1.1 Time, Complexity, and Cost of Testing Techniques. Common security testing techniques usually require manual interaction from security experts. In IoT, the complexity of the cybersecurity certification could require extensive and expensive efforts, which may delay the product launch and impact negatively the manufacturer from a competitiveness point of view. For that reason, there is a need to provide integrated security testing approaches throughout the lifecycle of an IoT system [74] based on efficient and automated techniques [117].

5.1.2 Frequent Security Updates. The security testing methodology should be able to manage the frequent changes associated to the security level of a product. This is especially relevant in the case of IoT, where frequent patching/update operations should be performed without the involvement of end-users. Indeed, the process of software updates represents an increasingly relevant topic in IoT scenarios. For example, the Software Updates for Internet of Things (SUIT) ${ }^{14}$ is an IETF initiative to adopt a firmware update solution by also considering systems and devices with tight resource constraints.

\footnotetext{
${ }^{14}$ https://datatracker.ietf.org/group/suit/about/.
} 
5.1.3 Scalability of Testing Techniques. The large amount of IoT system models to be certified requires the design of cost-effective testing procedures. Ideally, these techniques should be applicable to different types of systems and devices in such a way that similar procedures could serve to certify the security level of different components. Scalability could also be analyzed from a different perspective; indeed, it should be a core aspect of security testing techniques by simulating real-world IoT scenarios to detect possible security breaches of IoT devices (e.g., due to a DoS attack).

On the basis of previous considerations, we describe the current security testing approaches by using a classification that is defined in the next subsection.

\subsection{Categorizing Security Testing}

There are several features that can be used to classify the different testing approaches [22]. One of the mostly used aspects is the knowledge the tester has about the SUT. In black-box-testing, internal details of the SUT are not used, whereas in white-box-testing, SUT's internal details (e.g., source code) are considered. In terms of the execution, the testing procedure can be performed manually, so the human interaction guides the process, or automated, in which the tests are executed or generated through the use of specialized tools. Regarding the procedure, the testing can be performed dynamically against the running SUT, or statically, in which the execution of the SUT is not required. Concerning the test generation, it can be performed off-line if all the tests are generated before they are executed, or on-line, in which each test is generated and executed successively. An additional aspect is the focus, which can be represented by the attacker or the SUT itself. Other potential aspects to be considered are the test requirements and the test definition language. The former could be related to functional aspects (e.g., linked to the system functionality) or non-functional features (e.g., related to usability or performance) [121]. The latter could be defined in an informal (e.g., textual), semi-formal (e.g., UML), or formal way (e.g., through a logic-based language).

It should be noted that in this work, we consider the following security testing approaches: model-based testing, regression testing, code-based testing, penetration testing, fuzzing testing, dynamic taint, as well as different techniques or methods associated to each of them. Based on it, Table 4 in the Appendix shows the different testing approaches considered, which are classified following the selected criteria. Below, we provide a more detailed description of the main aspects of the different security testing approaches by using previous challenges as reference. A summarized description is also provided in Table 5 in the Appendix.

\subsection{Model-Based Testing (MBT)}

The main idea of Model-Based Testing (MBT) [36] is the usage of models, which could represent the test requirements, the SUT itself, and its environment. Based on it, Model-Based Security Testing (MBST) [73] aims to validate the security behavior of a certain software system considering a set of security properties. The model is usually represented as a high-level view of the SUT; consequently, there must be an additional process to adapt the abstract model into a real implementation of the SUT. Towards this end, the methodology makes use of an adapter, which represents an interface to implement the real tests to be executed on the SUT. The MBT (and MBST) process usually comprises several steps. First, a model is created to represent the SUT based on a set of testing requirements. For this, formal languages, such as UML, proprietary languages [190], or Domain Specific Languages (DSL) [59] are usually employed. Second, a set of implementation-independent tests are specified and generated based on the model previously generated. Third, such abstract tests are transformed into runnable tests through the use of adapters. Fourth, the adapted tests are executed manually or automatically on an execution environment. In the case of IoT, it could be 
represented by FIT-IoT, ${ }^{15}$ where large-scale deployments can be simulated. Finally, test results are collected to detect flaws and non-satisfactory test verdicts. Then, these results are used to ensure the conformance with the requirements specified at the beginning of the process.

One advantage of MBT and MBST is the possibility of automatically generating the model tests. In fact, currently there is a high number of MBT-based tools [111] that are intended to provide such automated approach (e.g., CertifyIt [110] or MISTA ${ }^{16}$ ). Due to the still-fragmented landscape of technologies, this aspect is especially relevant in the IoT context to represent a certain SUT independently of the underlying technology or protocol. However, the model definition and the requirements specification are manually performed. Taking into account the generation of the tests, on-line testing avoids the generation of all the tests before the execution, so the potentially high number of tests can be reduced. Furthermore, the generation of each test could be guided by including the results from the already executed tests.

Based on MBT, there are two perspectives. On the one hand, Behavioral MBT [36] focuses on modeling the behavior of the SUT by specifying the operations and entities involved on it. In this approach, the correct behavior is modeled, and it is used to generate test cases or to check a faulty behavior at runtime [181]. On the other hand, if the model is focused on modeling a malicious attack, it is called Attack patterns MBT [38]. In this approach, the pattern describes the goal, individual actions, conditions, and post-conditions of the attack to be implemented. Another classification of MBT approaches can be found in Reference [73].

\subsection{Regression Testing}

The main objective of regression testing [193] is to verify that any change on a certain SUT does not cause side effects, so that the system provides the intended functionality according to its specified requirements. This testing approach is necessary when there is a change in such requirements, or the software is modified according to new requirements through an updated/patching process. This is especially relevant for IoT scenarios; indeed, regression testing is applicable when a new vulnerability is discovered in a certain system or because of a change in the requirements that could need a security patch. In addition to the manufacturing phase, it can be applied during the operation/update phase of the IoT system lifecycle.

Regarding the already-mentioned classification aspects, regression testing could be realized by white-box and black-box approaches that can be performed manually or with the help of a tool that facilitates the automatic test classification and/or generation. An example is the tool proposed by Reference [78], which represents an extension of the tool CertifyIt [110]. As in the case of MBT, the approach can be on-line or off-line, depending on the tests generation.

There are several methodologies for regression testing [75], depending on the test coverage. In the case of test all, all the existing cases are executed, so it could imply an expensive and complex process. To cope with these aspects, other techniques try to optimize the testing procedures. For instance, Minimization is intended to remove some tests according to specific criteria. Furthermore, Prioritization orders the different tests by following a certain property, and Selection is employed to select a subset of tests, so only modified pieces of the software are actually tested.

\subsection{Code-based Testing}

Code-based testing [60] is a white-box testing approach to detect vulnerabilities and faults by using the source code of a system or application. Code reviews can be done manually, in which an expert reads the code [51], or automated, which is usually called Static Application

\footnotetext{
${ }^{15} \mathrm{https} / / \mathrm{www}$. iot-lab.info/.

${ }^{16} \mathrm{http}: / / \mathrm{cs}$. boisestate.edu/ dxu/research/MBT.html.
} 
Security Testing (SAST) [20]. For the latter, a SAST tool analyzes the source code of a certain component to report potential security problems through a syntax-checking process. Based on the understanding of data and control flows, semantic checks can also be used. Indeed, SAST tools are intended to analyze the different flows of a software to cope with the cumbersome task associated to manual source code reviews. Based on that, these tools can provide detailed recommendations to fix security issues very early in the development process. Compared to dynamic testing approaches, this technique provides a higher coverage of the SUT and a lower false negative rate (when a test passes but a threat is present). However, SAST tools are able to detect only vulnerabilities that were previously defined; thus, there is still a need for experts to configure properly the SAST tool to identify such vulnerabilities.

\subsection{Penetration Testing}

Penetration testing [30] is a testing approach where real-world attacks are simulated to determine methods for bypassing the security features provided by a SUT. While this approach is typically intended to test missing functionality or side-effects of the SUT, the target could also be the environment of the system (e.g., by exploiting an obsolete operating system). According to the described classification, penetration testing can be black-box if the attacker has limited information about the system or white-box if internal details are known. Furthermore, although penetration testing is often performed manually, there are tools helping the tester to discover weaknesses in a more automated way. This is the case of port or vulnerability scanners [28], which are used to discover security issues in applications. The scanner is used to query the interfaces of the application with various predefined attacks. Then, the application's responses are analyzed to know if the attack was realized, or at least, to get some information to make it successful.

\subsection{Fuzzing Testing and Dynamic Taint}

Fuzzing testing [171] is intended to test potential security flaws by using unexpected or incorrect inputs. On the one hand, it can be applied to the input data (data fuzzing testing [47]) by using random data as an input for the SUT to detect potential errors or vulnerabilities. On the other hand, it can be used as behavioral fuzzing testing $[160,176]$, in which valid/invalid message sequences are used for the same purpose. Fuzzing testing does not require the knowledge of the SUT implementation details, and consequently, it is considered a black-box testing method. Like in MBT and penetration testing, the behavioral fuzzing testing can be on-line or off-line, depending on the test generation. In the case of data fuzzing testing, the approach is usually realized off-line, since tests are generated before executing.

A fuzzing technique relies on its specific fuzz generator or fuzzer, which represents the algorithm in charge of fuzzing according to different strategies. The most basic approach of fuzzing testing is based on test data randomly generated (random fuzzing [47]). However, other more advanced approaches exist, such as mutation-based fuzzing testing [126], the model-based mutation testing [105], the model inference assisted (MIA) evolutionary fuzzing testing [64], or the approach proposed by Reference [37], in which MBT and fuzzing techniques are combined. Despite the high effectiveness of this technique and the ability to find zero-day vulnerabilities, a specific fuzzer must be implemented for each SUT. In addition, the number of generated tests must be limited according to a certain parameter or property. These aspects could represent significant scalability barriers especially in the case of the IoT landscape. To improve fuzzing testing, the objective of dynamic taint [74] is to mark specific data (e.g., coming from an untrusted source) as tainted. Then, it can be used to check how these tainted data are employed by the SUT to identify insecure data flows. In opposition to static analysis, dynamic taint analysis is conducted in a transparent way during the execution of the software in the SUT. This technique can be used in combination with fuzzing 


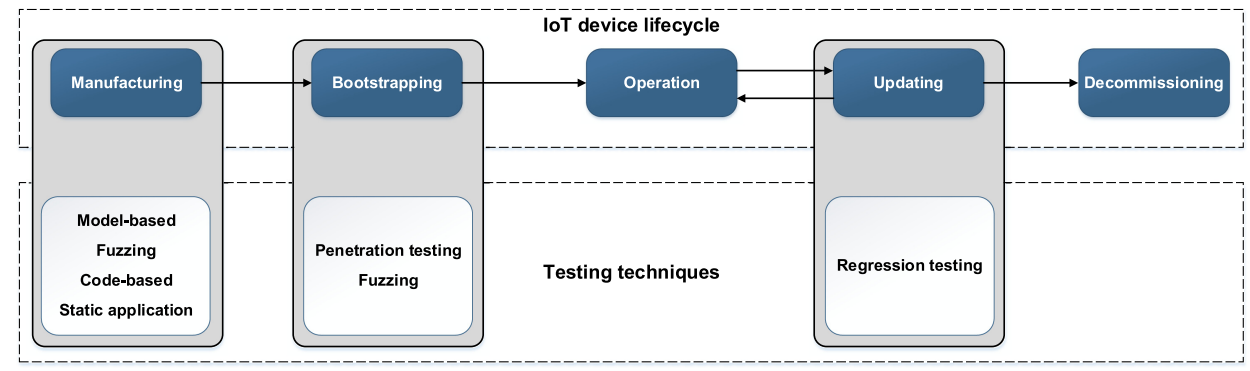

Fig. 3. Testing techniques according the IoT device lifecycle.

testing [29] to obtain information about the path execution by selecting the most promising test sequences to identify potential vulnerabilities.

\subsection{Security Testing: Analysis and Applicability to the loT Context}

Typically, there is not a single testing technique that fits in any scenario or situation. Indeed, according to References [74] and [63], different techniques and tools are actually required for security testing throughout the secure software development lifecycle. Based on the IoT device lifecycle described in Section 2.1, Figure 3 shows the relationship between the lifecycle of an IoT device and the application of different testing strategies. In general, during manufacturing, MBT can be used to provide conformance with security and functional requirements by following a security-by-design approach. Furthermore, static techniques can be used to review the code looking for security flaws, and fuzzing testing could be useful to analyze the behavior of the SUT against invalid data or message sequences before the device launch. Then, during bootstrapping, black-box strategies such as penetration testing are adequate to emulate a real attack and observe how the system reacts. In this case, data fuzzing could also provide relevant results by providing erroneous data to the SUT. Furthermore, in case of updating the device, regression testing can help to optimize the testing process to avoid to test the whole system. It should be noted that the different techniques can also be applied on different stages. For example, if initial functionality or requirements are modified during an update process, the use of MBT could be required to model the new version of the system. Indeed, for this situation, the use of MBT could help to avoid to test the whole system, in case regression testing is not enough. Below, we briefly describe recent works related to the application of different security techniques. The summary Table 6 is provided in the Appendix.

Regarding the application of MBT and MBST, we proposed their application for IoT security testing in previous works [4]. In particular, Reference [24] provides an initial description about their use as part of the certification and labeling processes of IoT devices as part of the Horizon 2020 ARMOUR project. ${ }^{17}$ This work is extended by Reference [140], in which the integration with a policy-based infrastructure is proposed by following the model-based security toolkit called Seckit [141]. Based on the ARMOUR approach, we provided an exhaustive practical approach in Reference [119] in which the use of MBT techniques is realized through UML and the Object Constrained Language (OCL) [40], which is employed to specify the system behavior under different test purposes. MBT is also considered by Reference [59], which is focused on the aircraft domain through the creation of a domain-specific language that is adapted to airplanes with the intention to detect false data injection attacks. Moreover, Reference [35] is focused on cryptographic components by using a subset of the UML/OCL notation. A similar approach [175] uses MBT with

\footnotetext{
${ }^{17}$ https://www.armour-project.eu/.
} 
OWL-S [26] notation to model the SUT as a finite state machine. Then, the test generation process is automated by using TTCN-3 [170] notation, as part of a creation environment for IoT. Furthermore, Reference [5] proposes to combine MBT with a service-oriented solution that is called MBTAAS. This approach is presented for testing data and IoT solutions, with experimentation on the European platform FIWARE. ${ }^{18}$ Moreover, Reference [173] uses model learning to derive models from different implementations of the same system that are compared to find vulnerabilities. Then, Reference [49] describes an MBT-based testing process of the Constrained Application Protocol (CoAP) [165], which is the standard application layer to be used in IoT scenarios. For this purpose, the authors of the paper develop a verification tool based on TTCN3 for automating the process. Additionally, Reference [172] uses an MBT approach to model both the behavior of the attacks and the SUT itself to generate tests. Then, they evaluate the analysis results of their model on a real smart-meter scenario.

Regarding static application testing, Reference [50] develops a tool (called DTaint) to scan taintstyle vulnerabilities in embedded firmware. Furthermore, authors apply such tool to different firmware images from several manufacturers to prove the effectiveness of their approach. Static analysis is also employed by Reference [57] to carry out a large-scale analysis of different embedded firmwares' security. Authors discovered 38 new vulnerabilities over 693 images and correlated firmware images to extend such vulnerabilities to other products.

In the case of the application penetration testing, Reference [7] proposes to use vulnerability scanning to test different IoT devices by using a specific IoT search engine called Shodan [33]. In Reference [32], penetration testing is used to analyze the security of different IP cameras. Furthermore, Reference [196] employs different phantom devices to mimic a a real system to detect new vulnerabilities in the scope of smart-home platforms. Also related to smart-home scenarios, Reference [12] analyzes the security of different devices against MITM attacks using an integration of different tools to automate the process for intercepting traffic. The approach proposed by Reference [52] is intended to find vulnerabilities and privacy violations, through manual code review and penetration testing, by using tools such as port scanners. The reported results demonstrate the lack of security and privacy best practices in the Smart Toys domain [174]. Moreover, Reference [101] proposes a security testbed framework based on the use of penetration testing techniques (e.g., through port and vulnerability scanning, fingerprinting) to simulate contextual conditions in which IoT devices operate. In particular, they apply their approach to smart-watch devices as an example of the Wearable IoT [89]. Furthermore, the thesis presented in Reference [148] proposes an approach to scan vulnerabilities of IoT devices being deployed in a certain network or system. For this purpose, authors propose the use of penetration testing techniques, even before the device is authorized to communicate with other devices or services in the network. Therefore, in case a vulnerability or threat is identified, the system tries to fix it; then, if it fails, the user is alerted. Moreover, Reference [158] describes an approach for analyzing the security of IoT devices through port scanning, fingerprinting, process enumeration, and vulnerability scanning. Then, after completing the penetration tests, the security analysis is carried out. In addition, Reference [182] proposes a tool called PENTOS to automatically obtain the information associated to a certain IoT device. PENTOS allows to perform different attacks (including wireless communication attacks), and it can retrieve reported vulnerabilities from existing repositories. More focused on specific IoT protocols, Reference [100] proposes to use the penetration testing tool Metasploit [120] to test the 6LoWPAN standard [131] against DoS attacks.

Moreover, fuzzing testing has also attracted the interest in the IoT ecosystem. In particular, Reference [15] is focused on IoT application-layer protocols by building a set of possible inputs

$\overline{{ }^{18} \text { https://www.fiware.org/. }}$ 
in each state of the protocol state machine. Towards a more general application, Reference [48] designs a fuzzing framework for discovering memory corruption vulnerabilities in IoT devices. Specifically, they reuse program-specific logic to mutate the test cases without the need of any knowledge about the underlying protocol specifications. Furthermore, Reference [194] develops a tool (FIRM-AFL) to enable grey-box fuzzing testing in emulated IoT firmwares. The approach is intended to cope with compatibility and performance issues associated to typical fuzzing systems. In Reference [184], authors develop a web-based tool (WMIFuzzer) to apply fuzzing testing to IoT devices. Their approach is based on a brute-force process to generate messages automatically and a mutation-based algorithm to generate structure valid messages. Fuzzing testing is also considered in Reference [186] to discover new vulnerabilities by mutating test cases that represent a valid attack path for a vulnerability. Moreover, in Reference [123], different CoAP implementations are tested through several fuzzing testing techniques by modeling a CoAP packet. Zigbee technology is tested by Reference [62] with a new fuzzing technique based on finite state machines. To this end, each state of the Zigbee device is tested through a tool called ZFSM-fuzzer. 6LoWPAN is also tested by Reference [107] through mutation fuzzing techniques and the use of the Scapy library [129]. For this purpose, each protocol is modelled through XML to specify different security testing aspects. Furthermore, Reference [132] designs a tool to test network protocol implementations. Such approach modifies the intended behavior of the protocol by using fuzzing testing techniques. Similarly, in Reference [168] an automated fuzz testing framework is designed to test IoT protocols in different stages of the secure software development lifecycle.

According to the previous analysis, Behavioral MBT and Penetration techniques represent popular approaches in the IoT paradigm. One of the main advantages of these approaches is the high amount of tools that can help to automate the process. Indeed, most of the related research proposals make use of additional tools to automate the process of discovering vulnerabilities. This fact highlights the importance for the authors of automating the testing process as much as possible. MBT-related techniques represent a promising alternative due to the possibility of generating tests from the SUT model in an automated way. This is reflected in the increasing interest of the research community. Indeed, we have used MBT in the scope of the Horizon 2020 ARMOUR project, ${ }^{19}$ in which the CertifyIt tool was employed to automate the testing process [119]. However, MBT still has to deal with the issue associated to the link between the high-level test specification and the real implementation of the TOE. This aspect could hinder the adoption of MBT if the implemented tests cannot be automatically generated from the model of the TOE. In spite of its advantages, and following the recommendations from other researchers [74], we claim that a security testing approach for IoT must embrace different techniques according to the lifecycle of the device or product. Such techniques will have to cope with different challenges, such as the replication of the physical environment where a certain device is deployed. This is particularly difficult in the IoT context, in which such environment could be changing due to the mobility of IoT devices and components.

\section{RECOMMENDATIONS AND POTENTIAL WAYS FORWARD}

This section summarizes the key findings from the previous sections and proposes potential ways forward to improve the IoT cybersecurity certification process.

- Cybersecurity certification should not stop with the initial certification, but it should support the entire lifecycle of the IoT product. This means that the process for re-evaluation and re-certification should be made more efficient in the highly dynamic IoT context where

\footnotetext{
${ }^{19}$ https://www.armour-project.eu/.
} 
the update of IoT devices can be quite frequent. As suggested in the previous sections, the use of MBT could support an effective regression testing and the identification of the boundaries of the update to limit the scope of the re-evaluation. As described in Section 5.8, the combination of MBT and TTCN3 can automate the re-evaluation, thus lowering time and costs. The link between the modelling of the TOE (using MBT) and the high-level security requirements could still be challenging for complex IoT systems, and further research is needed to refine the model or to introduce formalism, which can provide an indication on the model coverage.

- The fragmented landscape of cybersecurity certification schemes produces a disharmonized perspective for security evaluation. One of the main issues is lack of a unified format to represent the results of the certification process that could differ between different schemes. Furthermore, such schemes do not consider the use of machine-readable information that could help in a secure and automated deployment of devices. This is particularly relevant in the IoT context where non-expert users should be able to manage their devices. In this direction, the recent Manufacturer Usage Description (MUD) [108] could help to mitigate this issue through the definition of the intended purpose of a certain IoT device.

- Cybersecurity certification is considered as a key instrument to increase users' trust in the next digital era that requires the cooperation among different stakeholders, such as endusers, manufacturers, and institutions from different countries. To support such a collaboration, the establishment of a common platform to make such information accessible is key. Such platform should also serve as a repository of vulnerabilities that can be detected throughout the lifecycle of IoT devices by considering existing databases, such as the NVD in the U.S.

- The label concept can improve the transparency of the results of the cybersecurity certification process to the end-users both (a) to harmonize the presentation of the results across different products and (b) to simplify the complex jargon usually adopted in cybersecurity certification processes. We argue that the use of the label can be extended even further to support an effective cybersecurity monitoring of IoT infrastructures where the monitoring systems (e.g., intrusion detection) can exploit the information provided by the label and the underlying IoT device model. Further research is needed to support the design of monitoring systems based on the label concept.

- One challenge on the extension of the label concept to large IoT infrastructures is related to the problem of the composition of security properties. Further research is needed to revise the problem of security composition for its applicability to the label concept. A full or partial resolution of this problem could facilitate the cybersecurity certification of complex IoT system on the basis of the certification and labelling of the single components. In this direction, the use of hardware-software co-verification methods could be considered as part of the overall certification process of composed devices or systems [114]. However, these techniques need to be further analyzed in the coming years to check their suitability in the context of IoT.

- Testing procedures are essential for the certification process in most of cybersecurity certification schemes. However, there is a lack of standard definition of test sets that need to be passed for each assurance level and domain. The definition of such test sets would foster the harmonization of the certification results and the comparison between different devices. In this direction, a starting point could be the test plan proposed by the Cellular Telecommunications and Internet Association in the U.S. [61].

- As described in Section 4.9, there are various risk assessment processes defined in standardization bodies and research literature. However, many of them still have elements of 
subjectivity. We propose further research in the definition of rigorous risk assessment processes, which can be linked to model-based representation of the context and to the automated testing processes. A major focus of future research could be the definition of metrics, which can provide indication on the amount of risk coverage provided by the testing and assessment processes.

- Fuzzing testing is still not part of cybersecurity certification standards. We advocate a broader use and the fuzzing testing to increase the coverage of cybersecurity certification. As described in Section 5.7, fuzzing testing can be expanded using various variants, including behavioral fuzzing testing or mutation-based fuzzing testing.

\section{CONCLUSION}

Cybersecurity has traditionally represented one of the main concerns in ICT, and it is even more relevant in some domains like IoT. With the recent support of institutions, policy makers, and research community, certification is intended to provide a harmonized umbrella for cybersecurity implications. In particular, the recent Cybersecurity Act in Europe has triggered the need for defining consensual strategies to satisfy all the involved stakeholders. This survey provided a comprehensive overview of the main cybersecurity certification schemes, which were analyzed based on a set of defined requirements focused on the IoT paradigm. From the analysis, we concluded current approaches do not provide a suitable strategy for IoT cybersecurity certification. Based on recent institutional and research efforts, as well as the interest from manufacturers and policy makers, we focused on security risk assessment and testing as the main processes for the definition of such a framework. From our own experience, we claim the automation of both processes is key for the success of the framework. However, typical mechanisms have not demonstrated their suitability to cope with the challenges associated to the IoT ecosystem, in terms of scale, heterogeneity, and the need to address the cybersecurity certificate's lifecycle. While there is no silver-bullet scheme, this survey was intended to represent an initial effort to increase the awareness on the need to define aligned approaches to guide the realization of the mentioned framework in the coming years.

\section{REFERENCES}

[1] European Parliament. 2016. Directive 2010/41/EU of the European Parliament and of the Council of 7 July 2010. Retrieved from https://eur-lex.europa.eu/legal-content/EN/TXT/PDF/?uri=CELEX:32016L1148\&from=EN.

[2] Habtamu Abie and Ilangko Balasingham. 2012. Risk-based adaptive security for smart IoT in eHealth. In Proceedings of the 7th International Conference on Body Area Networks. ACM. DOI:https://doi.org/10.4108/icst.bodynets.2012. 250235

[3] Mohamed Abomhara and Geir M. Koien. 2015. Cyber security and the Internet of Things: Vulnerabilities, threats, intruders and attacks. F. Cyber Secur. Mobil. 4, 1 (2015), 65-88. DOI : https://doi.org/10.13052/jcsm2245-1439. 414

[4] Abbas Ahmad, Gianmarco Baldini, Philippe Cousin, Sara N. Matheu, Antonio Skarmeta, Elizabeta Fourneret, and Bruno Legeard. 2017. Cognitive Hyperconnected Digital Transformation: Internet of Things Intelligence Evolution. River Publishers, 189-220. Retrieved from https://books.google.es/books?id=nPIxDwAAQBAJ.

[5] Abbas Ahmad, Fabrice Bouquet, Elizabeta Fourneret, Franck Le Gall, and Bruno Legeard. 2016. Model-based testing as a service for IoT platforms. In Proceedings of the International Symposium on Leveraging Applications of Formal Methods. 727-742. DOI : https://doi.org/10.1007/978-3-319-47169-3_55

[6] AIOTI. 2016. Report on Workshop on Security and Privacy in the Hyper-Connected World. Retrieved from https://goo.gl/KeKqbs.

[7] Haneen Al-Alami, Ali Hadi, and Hussein Al-Bahadili. 2017. Vulnerability scanning of IoT devices in Jordan using Shodan. In Proceedings of the 2nd International Conference on the Applications of Information Technology in Developing Renewable Energy Processes and Systems (IT-DREPS'17). DOI : https://doi.org/10.1109/IT-DREPS.2017.8277814

[8] A. Al-Fuqaha, M. Guizani, M. Mohammadi, M. Aledhari, and M. Ayyash. 2015. Internet of Things: A survey on enabling technologies, protocols, and applications. IEEE Commun. Surv. Tutor. 17, 4 (2015), 2347-2376. DOI: https://doi.org/10.1109/COMST.2015.2444095 
[9] Christopher J. Alberts, Audrey J. Dorofee, James F. Stevens, and Carol Woody. 2005. OCTAVE-S Implementation Guide, Version 1. Technical Report. Carnegie Mellon University. Retrieved from https://resources.sei.cmu.edu/asset_files/ Handbook/2005_002_001_14273.pdf.

[10] Bako Ali and Ali Awad. 2018. Cyber and physical security vulnerability assessment for IoT-based smart homes. Sensors 18, 3 (Mar. 2018), 817. DOI : https://doi.org/10.3390/s18030817

[11] Lautenbach Aljoscha and Mafijul Islam. 2016. HEAling Vulnerabilities to ENhance Software Security and SafetyProject Proposal (HAVENS). Retrieved from http://autosec.se/wp-content/uploads/2018/03/HEAVENS_D2_v2.0.pdf.

[12] Omar Alrawi, Chaz Lever, Manos Antonakakis, and Fabian Monrose. 2019. SoK-Security evaluation of home-based IoT deployments. In Proceedings of the IEEE Symposium on Security and Privacy (SP'19). IEEE, 1362-1380. DOI: https://doi.org/10.1109/SP.2019.00013

[13] Faisal Alsubaei, Abdullah Abuhussein, and Sajjan Shiva. 2017. Security and privacy in the Internet of Medical Things: Taxonomy and risk assessment. In Proceedings of the IEEE 42nd Conference on Local Computer Networks Workshops (LCN Workshops'17). IEEE, 112-120. DOI : https://doi.org/10.1109/LCN.Workshops.2017.72

[14] Faisal Alsubaei, Abdullah Abuhussein, and Sajjan Shiva. 2018. Quantifying security and privacy in Internet of Things solutions. In Proceedings of the IEEE/IFIP Network Operations and Management Symposium (NOMS'18). IEEE, 1-6. DOI : https://doi.org/10.1109/NOMS.2018.8406318

[15] Prashant Anantharaman, Michael Locasto, Gabriela F. Ciocarlie, and Ulf Lindqvist. 2017. Building hardened Internetof-Things clients with language-theoretic security. In Proceedings of the IEEE Security and Privacy Workshops (SPW'17). IEEE, 120-126. DOI : https://doi.org/10.1109/SPW.2017.36

[16] Ross Anderson and Shailendra Fuloria. 2009. Certification and evaluation: A security economics perspective. In Proceedings of the IEEE Conference on Emerging Technologies \& Factory Automation. IEEE, 1-7. DOI : https://doi.org/ 10.1109/ETFA.2009.5347129

[17] ANSSI. 2008. Certification de Sécurité de Premier Niveau (CSPN). Retrieved from https://www.ssi.gouv.fr/ administration/produits-certifies/cspn/.

[18] ANSSI. 2018. Certification de Sécurité de Premier Niveau des Produits des Technologies de l'Information. Retrieved from https://www.ssi.gouv.fr/uploads/2015/01/anssi-cspn-cer-p-01-certification_de_securite_de_premier_niveau_ v2.0.pdf.

[19] Qazi Mamoon Ashraf and Mohamed Hadi Habaebi. 2015. Autonomic schemes for threat mitigation in Internet of Things. f. Netw. Comput. Applic. 49 (Mar. 2015), 112-127. DOI : https://doi.org/10.1016/j.jnca.2014.11.011

[20] Nathaniel Ayewah, David Hovemeyer, J. David Morgenthaler, John Penix, and William Pugh. 2008. Using static analysis to find bugs. IEEE Softw. 25, 5 (Sept. 2008), 22-29. DOI : https://doi.org/10.1109/MS.2008.130

[21] Hans Baars, Robert Lassche, Robin Massink, and Hans Pille. 2014. Smart grid security certification in Europe. Challenges and recommendations. Retrieved from https://www.enisa.europa.eu/publications/smart-grid-securitycertification-in-europe/at_download/fullReport.

[22] Ruediger Bachmann and Achim D. Brucker. 2014. Developing secure software. Datensch. Datensich. - DuD 38, 4 (Mar 2014), 257-261. DOI : https://doi.org/10.1007/s11623-014-0102-0

[23] Gianmarco Baldini, Georgios Giannopoulos, and Alessandro Lazari. 2017. Annex 8: JRC Analysis and Recommendations for a European Certification and Labelling Framework for Cybersecurity in Europe. Technical Report. European Commission. Retrieved from https:/ec.europa.eu/transparency/regdoc/rep/10102/2017/EN/SWD-2017-500-F1-ENMAIN-PART-6.PDF.

[24] Gianmarco Baldini, Antonio Skarmeta, Elizabeta Fourneret, Ricardo Neisse, Bruno Legeard, and Franck Le Gall. 2016. Security certification and labelling in Internet of Things. In Proceedings of the IEEE 3rd World Forum on Internet of Things (WF-IoT'16). IEEE, 627-632. DOI : https://doi.org/10.1109/WF-IoT.2016.7845514

[25] Aaron Ballman. 2016. SEI CERT C++ Coding Standard Edition: 98 Rules for Developing Safe, Reliable, and Secure Systems in C++. Retrieved from http://cysecure.org/455/dmccarroll/455/online/WeekTwo/Reading/sei-certcpp-coding-standard-2016-v01.pdf.

[26] Arthur Barstow, Mark Burstein, James Hendler, Vincent Marcatt, David Martin, Drew McDermott, Deborah L. McGuinness, Sheila McIlraith, Jeff Pollock, David De Roure, Mark Skall, Katia Sycara, and Hideki Yoshida. 2004. OWL-S-Semantic markup for Web services. W3C Member Submission 22 (2004). Retrieved from https://www. researchgate.net/publication/39994181_OWL-S_Semantic_markup_for_Web_services.

[27] Massimo Bartoletti, Pierpaolo Degano, and Gian Luigi Ferrari. 2006. Security issues in service composition. In Formal Methods for Open Object-based Distributed Systems, Vol. 4037. Springer Berlin, 1-16. DOI : https://doi.org/10.1007/ $11768869 \_1$

[28] Jason Bau, Elie Bursztein, Divij Gupta, and John Mitchell. 2010. State of the art: Automated black-box web application vulnerability testing. In Proceedings of the IEEE Symposium on Security and Privacy. IEEE, 332-345. DOI : https://doi. org/10.1109/SP.2010.27 
[29] Sofia Bekrar, Chaouki Bekrar, Roland Groz, and Laurent Mounier. 2011. Finding software vulnerabilities by smart fuzzing. In Proceedings of the 4th IEEE International Conference on Software Testing, Verification and Validation. IEEE, 427-430. DOI : https://doi.org/10.1109/ICST.2011.48

[30] Matt Bishop. 2007. About penetration testing. IEEE Secur. Priv. Mag. 5, 6 (Nov. 2007), 84-87. DOI : https://doi.org/10. 1109/MSP.2007.159

[31] BITAG. 2016. Internet of Things (IoT) Security and Privacy Recommendations. Retrieved from https://www.bitag. org/documents/BITAG_Report_-_Internet_of_Things_(IoT)_Security_and_Privacy_Recommendations.pdf.

[32] Kim Jonatan Wessel Bjørneset. 2017. Testing Security for Internet of Things. Survey on Vulnerabilities in IP Cameras. Ph.D. Thesis. University of Oslo. Retrieved from https:/www.mn.uio.no/ifi/english/research/groups/psy/ completedmasters/2017/Kim_Jonatan_Wessel_Bjorneset/kim_jonatan_wessel_bjorneset_testing_security_for_ internet_of_things_a_survey_on_vulnerabilities_in_ip_cameras.pdf.

[33] Roland Bodenheim, Jonathan Butts, Stephen Dunlap, and Barry Mullins. 2014. Evaluation of the ability of the Shodan search engine to identify Internet-facing industrial control devices. Int. F. Crit. Infrast. Protect. 7, 2 (June 2014), 114123. DOI : https://doi.org/10.1016/j.ijcip.2014.03.001

[34] Katie Boeckl, Michael Fagan, William Fisher, Naomi Lefkovitz, Katerina N. Megas, Ellen Nadeau, Danna Gabel O’Rourke, Ben Piccarreta, and Karen Scarfone. 2018. Considerations for Managing Internet of Things (IoT) Cybersecurity and Privacy Risks. https://doi.org/10.6028/NIST.IR.8228-draft

[35] Julien Botella, Fabrice Bouquet, Jean-Francois Capuron, Franck Lebeau, Bruno Legeard, and Florence Schadle. 2013. Model-based testing of cryptographic components-Lessons learned from experience. In Proceedings of the IEEE 6th International Conference on Software Testing, Verification and Validation. IEEE, 192-201. DOI : https://doi.org/10.1109/ ICST.2013.42

[36] F. Bouquet, C. Grandpierre, B. Legeard, F. Peureux, N. Vacelet, and M. Utting. 2007. A subset of precise UML for model-based testing. In Proceedings of the 3rd International Workshop on Advances in Model-based Testing (AMOST'07). ACM Press, 95-104. DOI : https://doi.org/10.1145/1291535.1291545

[37] Josip Bozic and Franz Wotawa. 2012. Model-based testing-From safety to security. In STV Bozic, Wotawa. 9-16. Retrieved from https://graz.pure.elsevier.com/en/publications/model-based-testing-from-safety-to-security.

[38] Josip Bozic and Franz Wotawa. 2014. Security testing based on attack patterns. In Proceedings of the IEEE 7th International Conference on Software Testing, Verification and Validation Workshops. IEEE, 4-11. DOI : https://doi.org/10. 1109/ICSTW.2014.58

[39] Miroslav Bures, Tomas Cerny, and Bestoun S. Ahmed. 2019. Internet of Things: Current challenges in the quality assurance and testing methods. In Proceedings of the International Conference on Information Science and Applications, Kuinam J. Kim and Nakhoon Baek (Eds.). Vol. 514. Springer Singapore, 625-634. DOI : https://doi.org/10.1007/978981-13-1056-0_61

[40] Jordi Cabot and Martin Gogolla. 2017. Object constraint language (OCL): A definitive guide. In Proceedings of the 12th International Conference on Formal Methods for the Design of Computer, Communication, and Software Systems: Formal Methods for Model-driven Engineering. DOI : https://doi.org/10.1007/978-3-642-30982-3_3

[41] Matteo Cagnazzo, Markus Hertlein, Thorsten Holz, and Norbert Pohlmann. 2018. Threat modeling for mobile health systems. In Proceedings of the IEEE Wireless Communications and Networking Conference Workshops (WCNCW'18). IEEE, 314-319. DOI : https://doi.org/10.1109/WCNCW.2018.8369033

[42] Richard A. Caralli, James F. Stevens, Lisa R. Young, and William R. Wilson. 2007. Introducing OCTAVE Allegro: Improving the Information Security Risk Assessment Process. Technical Report. CERT. Retrieved from https://resources.sei.cmu.edu/asset_files/TechnicalReport/2007_005_001_14885.pdf.

[43] CCRA. 2012. Common Criteria, Assurance Continuity, CCRA requirements. Version 2.1. Retrieved from http://www. commoncriteriaportal.org/files/operatingprocedures/2012-06-01.pdf.

[44] CCRA. 2017. Common Criteria for Information Technology Security Evaluation. Part 1: Introduction and general model.Retrieved from https://www.commoncriteriaportal.org/files/ccfiles/CCPART1V3.1R5.pdf.

[45] CERT SEI. 2018. Android Secure Coding Standard. Retrieved from https://wiki.sei.cmu.edu/confluence/display/ android/Android+Secure+Coding+Standard.

[46] CESG. 2014. The Commercial Product Assurance (CPA) build standard. Retrieved from https://www.ncsc.gov.uk/ content/files/protected_files/document_files/The\%20CPA\%20Build\%20Standard\%201.3.pdf.

[47] Chen Chen, Baojiang Cui, Jinxin Ma, Runpu Wu, Jianchao Guo, and Wenqian Liu. 2018. A systematic review of fuzzing techniques. Comput. Secur. 75 (June 2018), 118-137. DOI : https://doi.org/10.1016/j.cose.2018.02.002

[48] Jiongyi Chen, Wenrui Diaoy, Qingchuan Zhaoz, Chaoshun Zuoz, Zhiqiang Linz, XiaoFeng Wangx, Wing Cheong Lau, Menghan Sun, Ronghai Yang, and Kehuan Zhang. 2018. IoTFuzzer-Discovering memory corruptions in IoT through app-based fuzzing. In Proceedings of the Network and Distributed System Security Symposium. DOI: https://doi.org/10.14722/ndss.2018.23166 
[49] Nanxing Chen, César Viho, Anthony Baire, Xiaohong Huang, and Jiexi Zha. 2012. Ensuring interoperability for the Internet of Things: Experience with CoAP protocol testing. F. Contr. Meas. Electron. Comput. Commun. 6 (2012), 448-458. DOI : https://doi.org/10.7305/automatika.54-4.418

[50] Kai Cheng, Qiang Li, Lei Wang, Qian Chen, Yaowen Zheng, Limin Sun, and Zhenkai Liang. 2018. DTaint-Detecting the taint-style vulnerability in embedded device firmware. In Proceedings of the 48th Annual IEEE/IFIP International Conference on Dependable Systems and Networks (DSN'18). 430-441. DOI: https://doi.org/10.1109/DSN.2018.00052

[51] Brian Chess and Jabob West. 2007. Secure Programming with Static Analysis. Gary McGraw. Retrieved from https://www.e-reading.club/bookreader.php/142130/Secure_programming_with_Static_Analysis.pdf.

[52] Gordon Chu, Noah Apthorpe, and Nick Feamster. 2018. Security and privacy analyses of Internet of Things toys. IEEE Internet Things f. 6, 1 (2018), 978-985. DOI : https://doi.org/10.1109/JIOT.2018.2866423

[53] Peter Cihon, Glenda Michel Gutierrez, Sam Kee, Moritz Jan Kleinaltenkamp, Thanel Voigt, and Antonio Rosato. 2018. Why certify? Increasing adoption of the proposed EU cybersecurity certification framework. Cambridge Judge Business School, Sophia Antipolis, France. Retrieved from https://docbox.etsi.org/Workshop/2018/201806 ETSISECURITYWEEK/IoTSecurity/00POSTERS/Cambridge\%20EU\%20Cybersecurity\%20Certification\%20Report. pdf.

[54] Sara Cleemput. 2018. Secure and Privacy-friendly Smart Electricity Metering. Ph.D. Thesis. Arenberg Doctoral School. Faculty of Engineering Science. Retrieved from https:/www.esat.kuleuven.be/cosic/publications/thesis-303.pdf.

[55] CNSSI. 2015. CNSSI No. 4009: Committee on National Security Systems (CNSS) Glossary. Retrieved from https://cryptosmith.files.wordpress.com/2015/08/glossary-2015-cnss.pdf.

[56] Common Criteria. 2014. Arrangement on the Recognition of Common Criteria Certificates in the field of Information Technology Security. Retrieved from https:/www.commoncriteriaportal.org/files/operatingprocedures/ccrecarrange.pdf.

[57] Andrei Costin, Jonas Zaddach, Aurélien Francillon, and Davide Balzarotti. 2014. A large-scale analysis of the security of embedded firmwares. 95-110. Retrieved from https://www.usenix.org/node/184450.

[58] Antoine Coutant. 2016. French Scheme CSPN to CC evaluation. Retrieved from http://www.yourcreativesolutions nl/ICCC13/p/CC\%20and\%20New\%20Techniques/Antoine\%20COUTANT\%20-\%20CSPN\%20to\%20CC\%20Evaluation. pdf.

[59] Aymeric Cretin, Bruno Legeard, Fabien Peureux, and Alexandre Vernotte. 2018. Increasing the resilience of ATC systems against false data injection attacks using DSL-based testing. In Proceedings of the Doctoral Symposium (ICRAT'18).

[60] Lajos Cseppento and Zoltan Micskei. 2017. Evaluating code-based test input generator tools. Softw. Test. Verif. Reliab. 27, 6 (Sept. 2017), e1627. DOI : https://doi.org/10.1002/stvr.1627

[61] CTIA. 2018. Cybersecurity Certification Test Plan for IoT Devices. Retrieved from https://api.ctia.org/wp-content/ uploads/2018/08/CTIA-IoT-Cybersecurity-Certification-Test-Plan-V1_0.pdf.

[62] Baojiang Cui, Shurui Liang, Shilei Chen, Bing Zhao, and Xiaobing Liang. 2014. A novel fuzzing method for Zigbee based on finite state machine. Int. f. Distrib. Sensor Netw. 10, 1 (Jan. 2014), 762891. DOI : https://doi.org/10.1155/2014/ 762891

[63] Joao Pedro Dias, Flavio Couto, Ana C. R. Paiva, and Hugo Sereno Ferreira. 2018. A brief overview of existing tools for testing the Internet-of-Things. In Proceedings of the IEEE International Conference on Software Testing, Verification and Validation Workshops (ICSTW'18). IEEE, 104-109. DOI : https://doi.org/10.1109/ICSTW.2018.00035

[64] Fabien Duchene. 2014. Detection of Web Vulnerabilities via Model Inference assisted Evolutionary Fuzzing. Ph.D. Dissertation. Grenoble University. Retrieved from https://hal.archives-ouvertes.fr/tel-01102325/document.

[65] ECSO. 2017. A Meta-Scheme Approach v1.0. Retrieved from http://www.ecs-org.eu/documents/uploads/europeancyber-security-certification-a-meta-scheme-approach.pdf.

[66] ECSO. 2017. State of the Art Syllabus v2. Retrieved from http://www.ecs-org.eu/documents/uploads/updated-sota. pdf.

[67] ENISA. 2018. Overview of ICT certification laboratories. Retrieved from http://www.european-accreditation.org/ brochure/document-ict-certification-laboratories.

[68] Gencer Erdogan, Yan Li, Ragnhild Kobro Runde, Fredrik Seehusen, and Ketil Stølen. 2014. Approaches for the combined use of risk analysis and testing: A systematic literature review. Int. F. Softw. Tools Technol. Transf. 16 (2014), 627-642. DOI : https://doi.org/10.1007/s10009-014-0330-5

[69] ETSI. 2015. ETSI EG 203 251: Methods for Testing \& Specification; Risk-based Security Assessment and Testing Methodologies. Retrieved from https://www.etsi.org/deliver/etsi_eg/203200_203299/203251/01.01.01_50/eg_ 203251v010101m.pdf.

[70] European Commission. 2010. Directive 2010/30/EU on the indication by labelling and standard product information of the consumption of energy and other resources by energy-related products. Retrieved from http://eur-lex.europa. eu/legal-content/EN/TXT/?uri=CELEX:32010L0030. 
[71] European Parliament. 2016. REGULATION (EU) 2016/679 OF THE EUROPEAN PARLIAMENT AND OF THE COUNCIL of 27 April 2016 on the protection of natural persons with regard to the processing of personal data and on the free movement of such data, and repealing Directive 95/46/EC (General Data Protection Regulation). Retrieved from https://eugdpr.org/.

[72] EVITA. 2008. E-Safety Vehicle Intrusion Protected Applications. Retrieved from https://www.evita-project.org/.

[73] Michael Felderer, Berthold Agreiter, Philipp Zech, and Ruth Breu. 2011. A classification for model-based security testing. 109-114. Retrieved from https://www.thinkmind.org/index.php?view=article\&articleid=valid_2011_5_10_ 40020 .

[74] Michael Felderer, Matthias Büchler, Martin Johns, Achim D. Brucker, Ruth Breu, and Alexander Pretschner. 2015. Chapter one - Security testing: A survey. In Advances in Computers. Vol. 101. Elsevier, 1-51. DOI : https://doi.org/10. 1016/bs.adcom.2015.11.003

[75] Michael Felderer and Elizabeta Fourneret. 2015. A systematic classification of security regression testing approaches. Int. J. Softw. Tools Technol. Transf. 17, 3 (June 2015), 305-319. DOI : https://doi.org/10.1007/s10009-015-0365-2

[76] Michael Felderer and Ina Schieferdecker. 2014. A taxonomy of risk-based testing. Int. f. Softw. Tools Technol. Transf. 16, 5 (Oct. 2014), 559-568. DOI : https://doi.org/10.1007/s10009-014-0332-3

[77] FIRST. 2015. Common Vulnerability Score System (CVSS) v3. Retrieved from https://www.first.org/cvss/cvss-v30specification-v1.8.pdf.

[78] Elizabeta Fourneret, Fabrice Bouquet, Frederic Dadeau, and Stephane Debricon. 2011. Selective test generation method for evolving critical systems. In Proceedings of the IEEE 4th International Conference on Software Testing, Verification and Validation Workshops. IEEE, 125-134. DOI : https://doi.org/10.1109/ICSTW.2011.95

[79] Anna Baron Garcia, Radu F. Babiceanu, and Remzi Seker. 2018. Trustworthiness requirements and models for aviation and aerospace systems. In Proceedings of the Integrated Communications, Navigation, Surveillance Conference (ICNS'18). IEEE, 1-16. DOI : https://doi.org/10.1109/ICNSURV.2018.8384911

[80] Mengmeng Ge, Jin B. Hong, Walter Guttmann, and Dong Seong Kim. 2017. A framework for automating security analysis of the internet of things. F. Netw. Comput. Applic. 83 (Apr. 2017), 12-27. DOI : https://doi.org/10.1016/j.jnca. 2017.01.033

[81] Mengmeng Ge and Dong Seong Kim. 2015. A framework for modeling and assessing security of the Internet of Things. In Proceedings of the IEEE 21st International Conference on Parallel and Distributed Systems (ICPADS'15). 776-781. DOI : https://doi.org/10.1109/ICPADS.2015.102

[82] Gemini George and Sabu M. Thampi. 2018. A graph-based security framework for securing industrial IoT networks from vulnerability exploitations. IEEE Access 6 (2018), 43586-43601. DOI : https://doi.org/10.1109/ACCESS. 2018.2863244

[83] J. Granjal, E. Monteiro, and J. Sa Silva. 2015. Security for the Internet of Things: A survey of existing protocols and open research issues. IEEE Commun. Surv. Tutor. 17, 3 (2015), 1294-1312. DOI : https://doi.org/10.1109/COMST.2015. 2388550

[84] Jurgen Grossmann, Michael Felderer, Johannes Viehmann, and Ina Schieferdecker. 2019. A taxonomy to assess and tailor risk-based testing in recent testing standards. IEEE Softw. PP (May 2019), 1-1. DOI : https://doi.org/10.1109/MS. 2019.2915297

[85] GSMA. 2016. IoT Security Guidelines Overview Document. Retrieved from https://www.gsma.com/iot/wp-content/ uploads/2016/02/CLP.11-v1.1.pdf.

[86] Ayyoob Hamza, Dinesha Ranathunga, Hassan Habibi Gharakheili, Theophilus A. Benson, Matthew Roughan, and Vijay Sivaraman. 2019. Verifying and monitoring IoTs network behavior using MUD profiles. Retrieved from http://arxiv.org/abs/1902.02484.

[87] Wenxi Han, Xiaoming Liu, Hong Zhang, Ruijie Quan, and Linfeng Shen. 2018. Dynamically-enabled defense effectiveness evaluation of IoT based on vulnerability analysis. In Proceedings of the 3rd International Conference on Multimedia Systems and Signal Processing (ICMSSP'18). ACM Press, 99-103. DOI : https://doi.org/10.1145/3220162.3220170

[88] J. Hearn. 2004. Does the common criteria paradigm have a future?IEEE Secur. Priv. Mag. 2, 1 (Jan. 2004), 64-65. DOI : https://doi.org/10.1109/MSECP.2004.1264857

[89] S. Hiremath, G. Yang, and K. Mankodiya. 2014. Wearable Internet of Things: Concept, architectural components and promises for person-centered healthcare. In Proceedings of the 4th International Conference on Wireless Mobile Communication and Healthcare-Transforming Healthcare through Innovations in Mobile and Wireless Technologies (MOBIHEALTH'14). 304-307. DOI : https://doi.org/10.1109/MOBIHEALTH.2014.7015971

[90] Juliane Hubner and Maria Lastovka. 2017. BOSCH Political Viewpoint. Security in IoT. Retrieved from https://www. boschsecurity.com/xc/en/news/rethink-the-magazine/winds-of-change/.

[91] ICSA. 2016. ICSA Labs IoT Security and Privacy. Retrieved from https://www.icsalabs.com/technology-program/iotdevices-sensors/iot-device-requirements-framework. 
[92] ICSA. 2016. Internet of Things (IoT) Security Testing Framework. Retrieved from https://www.icsalabs.com/sites/ default/files/body_images/ICSALABS_IoT_reqts_framework_v2.0_161026.pdf.

[93] Information Technology Promotion Agency (IPA). 2019. Japan Information Technology Security Evaluation and Certification Scheme. Retrieved from https://www.ipa.go.jp/security/jisec/jisec_e/.

[94] IoT Security Fundation. 2017. IoT Security Compliance Framework. Release 1.1. Retrieved from https://www. iotsecurityfoundation.org/wp-content/uploads/2017/12/IoT-Security-Compliance-Framework_WG1_2017.pdf.

[95] ISO. 2018. Information technology-Internet of Things (IoT)-Vocabulary (ISO/IEC 20924:2018). Retrieved from http://www.iso.org/cms/render/live/en/sites/isoorg/contents/data/standard/06/94/69470.html.

[96] Andreas Jacobsson, Martin Boldt, and Bengt Carlsson. 2016. A risk analysis of a smart home automation system. Fut. Gen. Comput. Syst. 56 (Mar. 2016), 719-733. DOI : https://doi.org/10.1016/j.future.2015.09.003

[97] Joint Task Force Transformation Initiative. 2014. Guide for Applying the Risk Management Framework to Federal Information Systems: A Security Life Cycle Approach. Technical Report NIST SP 800-37r1. National Institute of Standards and Technology. DOI : https://doi.org/10.6028/NIST.SP.800-37r1

[98] Sathya Prakash Kadhirvelan and Andrew Soderberg-Rivkin. 2014. Threat Modelling and Risk Assessment within Vehicular Systems. Ph.D. Dissertation. University of Gothenburg. Retrieved from http://publications.lib.chalmers.se/ records/fulltext/202917/202917.pdf.

[99] Samuel Paul Kaluvuri, Michele Bezzi, and Yves Roudier. 2014. A quantitative analysis of common criteria certification practice. In Trust, Privacy, and Security in Digital Business. Vol. 8647. Springer International Publishing, Cham, 132143. DOI : https://doi.org/10.1007/978-3-319-09770-1_12

[100] Prabhakaran Kasinathan, Claudio Pastrone, Maurizio A. Spirito, and Mark Vinkovits. 2013. Denial-of-service detection in 6LoWPAN based Internet of Things. In Proceedings of the IEEE 9th International Conference on Wireless and Mobile Computing, Networking and Communications (WiMob'13). IEEE, 600-607. DOI : https://doi.org/10.1109/ WiMOB.2013.6673419

[101] Prabhakaran Kasinathan, Claudio Pastrone, Maurizio A. Spirito, Mark Vinkovits, Nils O. Tippenhauer Jemin Lee Shachar Siboni, Asaf Shabtai, and Yuval Elovici. 2016. Advanced security testbed framework for wearable IoT devices. In Proceedings of the IEEE 9th International Conference on Wireless and Mobile Computing, Networking and Communications (WiMob'13), Vol. 16. DOI : https://doi.org/10.1145/2981546

[102] Kaspersky. 2017. Kaspersky Labs Targeted Attacks Detection Solution Is Certified by ICSA Labs. Retrieved from https: //www.kaspersky.com/about/press-releases/2017_targeted-attacks-detection-solution-certified-by-icsa-labs.

[103] F. Keblawi and D. Sullivan. 2006. Applying the common criteria in systems engineering. IEEE Secur. Priv. Mag. 4, 2 (Mar. 2006), 50-55. DOI : https://doi.org/10.1109/MSP.2006.35

[104] Constantinos Kolias, Georgios Kambourakis, Angelos Stavrou, and Jeffrey Voas. 2017. DDoS in the IoT: Mirai and other botnets. Computer 50, 7 (2017), 80-84. DOI : https://doi.org/10.1109/MC.2017.201

[105] Willibald Krenn, Rupert Schlick, Stefan Tiran, Bernhard Aichernig, Elisabeth Jobstl, and Harald Brandl. 2015. MoMut-UML model-based mutation testing for UML. In Proceedings of the IEEE 8th International Conference on Software Testing, Verification and Validation (ICST'15). IEEE, 1-8. DOI : https://doi.org/10.1109/ICST.2015.7102627

[106] Ievgeniia Kuzminykh and Anders Carlsson. 2018. Analysis of assets for threat risk model in avatar-oriented IoT architecture. In Internet of Things, Smart Spaces, and Next Generation Networks and Systems (Lecture Notes in Computer Science), Olga Galinina, Sergey Andreev, Sergey Balandin, and Yevgeni Koucheryavy (Eds.). Springer International Publishing, Cham, 52-63. DOI : https://doi.org/10.1007/978-3-030-01168-0_6

[107] Abdelkader Lahmadi, Cesar Brandin, and Olivier Festor. 2012. A testing framework for discovering vulnerabilities in 6LoWPAN networks. In Proceedings of the IEEE 8th International Conference on Distributed Computing in Sensor Systems. IEEE, 335-340. DOI : https://doi.org/10.1109/DCOSS.2012.48

[108] Eliot Lear, Dan Romascanu, and Ralph Droms. 2019. Manufacturer Usage Description Specification (RFC 8520). Retrieved from https://tools.ietf.org/html/rfc8520.

[109] Seokcheol Lee, Sungjin Kim, Ken Choi, and Taeshik Shon. 2018. Game theory-based security vulnerability quantification for social Internet of Things. Fut. Gen. Comput. Syst. 82 (May 2018), 752-760. DOI : https://doi.org/10.1016/j. future.2017.09.032

[110] Bruno Legeard and Arnaud Bouzy. 2013. Smartesting CertifyIt: Model-based testing for enterprise IT. In Proceedings of the IEEE 6th International Conference on Software Testing, Verification and Validation. IEEE, 391-397. DOI : https: //doi.org/10.1109/ICST.2013.55

[111] Wenbin Li, Franck Le Gall, and Naum Spaseski. 2018. A survey on model-based testing tools for test case generation. In Tools and Methods of Program Analysis, Vladimir Itsykson, Andre Scedrov, and Victor Zakharov (Eds.), Vol. 779. Springer International Publishing, Cham, 77-89. DOI : https://doi.org/10.1007/978-3-319-71734-0_7

[112] Caiming Liu, Yan Zhang, Jinquan Zeng, Lingxi Peng, and Run Chen. 2012. Research on dynamical security risk assessment for the Internet of Things inspired by immunology. In Proceedings of the 8th International Conference on Natural Computation. IEEE, 874-878. DOI : https://doi.org/10.1109/ICNC.2012.6234533 
[113] Fred Long, Dhruv Mohindra, and Robert C. Seacord. 2011. The Cert Oracle Secure Coding Standard for fava (1st ed.). Addison Wesley Pub. Co. Inc., Upper Saddle River, NJ.

[114] Florian Lugou, Ludovic Apvrille, and Aurélien Francillon. 2016. Toward a methodology for unified verification of hardware/software co-designs. F. Cryptog. Eng. (Nov. 2016), 1-12. DOI : https://doi.org/10.1007/s13389-016-0145-2

[115] Imran Makhdoom, Mehran Abolhasan, Justin Lipman, Ren Ping Liu, and Wei Ni. 2018. Anatomy of threats to the Internet of Things. IEEE Commun. Surv. Tutor. (2018), 1-1. DOI: https://doi.org/10.1109/COMST.2018.2874978

[116] Mark Miller. 2018. D3.2 European cybersecurity and privacy Research and Innovation Ecosystem. Retrieved from https://www.cyberwatching.eu/sites/default/files/D3.2_European_cybersecurity_and_privacy_Research_ \%26Innovation_Ecosystem.pdf.

[117] S. N. Matheu, J. L. Hernandez-Ramos, and A. F. Skarmeta. 2019. Toward a cybersecurity certification framework for the Internet of Things. IEEE Secur. Priv. 17, 3 (May 2019), 66-76. DOI : https://doi.org/10/gf256z

[118] Sara N. Matheu-Garcia, Jose L. Hernandez-Ramos, and Antonio F. Skarmeta. 2018. Test-based risk assessment and security certification proposal for the Internet of Things. In Proceedings of the IEEE 4th World Forum on Internet of Things (WF-IoT'18). IEEE, 641-646. DOI : https://doi.org/10.1109/WF-IoT.2018.8355193

[119] Sara N. Matheu-Garcia, Jose L. Hernandez-Ramos, Antonio F. Skarmeta, and Gianmarco Baldini. 2019. Risk-based automated assessment and testing for the cybersecurity certification and labelling of IoT devices. Comput. Stand. Interf. 62 (Feb. 2019), 64-83. DOI : https://doi.org/10.1016/j.csi.2018.08.003

[120] David Maynor. 2011. Metasploit Toolkit for Penetration Testing, Exploit Development, and Vulnerability Research. Elsevier. Google-Books-ID: JWgNVFtbWJ4C. Retrieved from https://www.elsevier.com/books/metasploit-toolkit-forpenetration-testing-exploit-development-and-vulnerability-research/maynor/978-1-59749-074-0.

[121] G. Mcgraw. 2004. Software security. IEEE Secur. Priv. Mag. 2, 2 (Mar. 2004), 80-83. DOI: https://doi.org/10.1109/ MSECP.2004.1281254

[122] Kais Mekki, Eddy Bajic, Frederic Chaxel, and Fernand Meyer. 2019. A comparative study of LPWAN technologies for large-scale IoT deployment. ICT Express 5, 1 (Mar. 2019), 1-7. DOI : https://doi.org/10/gfsc2n

[123] Bruno Melo, Paulo Licio Geus, and Andre A. Gregio. 2017. Robustness testing of CoAP server-side implementations through black-box fuzzing techniques. In Proceedings of the Brazilian Symposium on Information Security and Computer Systems. 533-540. Retrieved from https://pdfs.semanticscholar.org/487b/ 7a45bc5962fd2cdf65da2caa05fcaef64591.pdf.

[124] Microsoft. 2018. The STRIDE Threat Model. Retrieved from https://msdn.microsoft.com/en-us/library/ee823878(v= cs.20).aspx.

[125] Microsoft. 2010. DREAD scheme. Retrieved from https://docs.microsoft.com/en-us/previous-versions/msp-n-p/ ff648644(v=pandp.10)\#dread.

[126] Charlie Miller and Zachary Peterson. 2007. Analysis of mutation and generation-based fuzzing. Retrieved from http://mirror.picosecond.org/defcon/defcon15-cd/Speakers/Miller/Whitepaper/dc-15-miller-WP.pdf.

[127] MITRE. 2011. Common Weakness Risk Analysis Framework (CWRAF). Retrieved from https://cwe.mitre.org/cwraf/.

[128] MITRE. 2014. CWE-Common Weakness Scoring System (CWSS). Retrieved from https://cwe.mitre.org/cwss/cwss_ v1.0.1.html.

[129] Robert Montante. 2018. Using Scapy in teaching network header formats: Programming network headers for nonprogrammers (abstract only). In Proceedings of the 49th ACM Technical Symposium on Computer Science Education (SIGCSE'18). ACM, New York, NY, 1106-1106. DOI : https://doi.org/10.1145/3159450.3162228

[130] K. Moore, R. Barnes, and H. Tschofenig. 2016. Best Current Practices for Securing Internet of Things (IoT) Devices. Retrieved from https://tools.ietf.org/html/draft-moore-iot-security-bcp- 00 .

[131] Geoff Mulligan. 2007. The 6LoWPAN architecture. In Proceedings of the 4th Workshop on Embedded Networked Sensors (EmNets'07). ACM, New York, NY, 78-82. DOI : https://doi.org/10.1145/1278972.1278992

[132] Tewodros Legesse Munea, I. Luk Kim, and Taeshik Shon. 2017. Design and implementation of fuzzing framework based on IoT applications. Wirel. Person. Commun. 93, 2 (Mar. 2017), 365-382. DOI : https://doi.org/10.1007/s11277016-3322-9

[133] Steven Murdoch, Mike Bond, and Ross J. Anderson. 2012. How certification systems fail: Lessons from the ware report. IEEE Secur. Priv. Mag. 10, 6 (2012), 1-1. DOI : https://doi.org/10.1109/MSP.2012.89

[134] National Cybersecurity Center of United Kingdom. 2017. Foundation Grade explained. Retrieved from https://www. ncsc.gov.uk/articles/foundation-grade-explained.

[135] National Cybersecurity Center (UK). 2016. CPA SC Overwriting Tools for Magnetic Media v2-1. Retrieved from https://www.ncsc.gov.uk/content/files/protected_files/document_files/CPA\%20SC\%20Overwriting\%20Tools\%20for \%20Magnetic\%20Media\%20v2-1.pdf.

[136] National Cybersecurity Centre (UK). 2016. Process for performing commercial product assurance foundation grade evaluations. Retrieved from https:/www.ncsc.gov.uk/content/files/protected_files/document_files/ Process $\% 20$ for\%20Performing\%20CPA\%20Foundation\%20Grade\%20Evaluations\%202-4.pdf. 
[137] NCC Group. 2016. Commercial Product Assurance and Common Criteria. Retrieved from https://www.nccgroup. trust/uk/our-services/cyber-security/compliance-and-accreditations/cpa-and-cc/.

[138] NCC Group. 2007. CERT C Programming Language Secure Coding Standard. Retrieved from http://www.open-std. org/jtc1/sc22/wg14/www/docs/n1255.pdf.

[139] NCC Group. 2016. Threat prioritisation: DREAD is dead, baby?Retrieved from https://www.nccgroup.trust/uk/ about-us/newsroom-and-events/blogs/2016/march/threat-prioritisation-dread-is-dead-baby/.

[140] Ricardo Neisse, Gianmarco Baldini, Gary Steri, Abbas Ahmad, Elizabeta Fourneret, and Bruno Legeard. 2017. Improving Internet of Things device certification with policy-based management. In Proceedings of the Global Internet of Things Summit (GIoTS'17). IEEE, 1-6. DOI : https://doi.org/10.1109/GIOTS.2017.8016273

[141] Ricardo Neisse, Gary Steri, Igor Nai Fovino, and Gianmarco Baldini. 2015. SecKit-A model-based security toolkit for the Internet of Things. Comput. Secur. 54 (Oct. 2015), 60-76. DOI : https://doi.org/10.1016/j.cose.2015.06.002

[142] NIST. 2019. Glossary of Key Information Security Terms. Retrieved from https://www.nist.gov/publications/glossarykey-information-security-terms-2.

[143] NIST. 2006. FIPS 200, Minimum Security Requirements for Federal Information and Information Systems. Retrieved from https://csrc.nist.gov/publications/detail/fips/200/final.

[144] NIST. 2014. Framework for Improving Critical Infrastructure Cybersecurity, Version 1.0. Retrieved from https:// nvlpubs.nist.gov/nistpubs/CSWP/NIST.CSWP.04162018.pdf.

[145] NIST. 2018. Framework for Improving Critical Infrastructure Cybersecurity, Version 1.1. Technical Report. National Institute of Standards and Technology. https://doi.org/10.6028\%2Fnist.cswp.04162018

[146] NIST. 2018. Risk Management Framework for Information Systems and Organizations. Retrieved from https://csrc. nist.gov/CSRC/media/Publications/sp/800-37/rev-2/draft/documents/sp800-37r2-draft-fpd.pdf.

[147] Jason R. C. Nurse, Sadie Creese, and David De Roure. 2017. Security risk assessment in Internet of Things systems. IEEE Computer Society, IT Pro (2017).

[148] Ruth Motunrayo Ogunnaike. 2017. Vulnerability Detection and Resolution in Internet of Things (IoT) Devices. Master Thesis. University of Washington.

[149] Adebayo Omotosho, Benjamin Ayemlo Haruna, and Olayemi Mikail Olaniyi. 2019. Threat modeling of Internet of Things health devices. F. Appl. Secur. Res. 14, 1 (Jan. 2019), 106-121. DOI: https://doi.org/10.1080/19361610.2019. 1545278

[150] Online Trust Alliance. 2017. IoT Security \& Privacy Trust Framework v2.5. Retrieved from https://otalliance.org/ system/files/files/initiative/documents/iot_trust_framework6-22.pdf.

[151] Openstack. 2014. Security/OSSA-Metrics. Retrieved from https://wiki.openstack.org/wiki/Security/OSSA-Metrics\# Calibration.

[152] OWASP. [n.d.]. OWASP Application Security Verification Standard (ASVS) Project. Retrieved from https://www.owasp. org/index.php/OWASP_Risk_Rating_Methodology.

[153] Euopean Parliament. 2019. Regulation (EU) 2019/881 of the European Parliament and of the council of 17 April 2019 on ENISA (the European Union Agency for Cybersecurity) and on information and communications technology cybersecurity certification (Cybersecurity Act). Retrieved from https://eur-lex.europa.eu/legal-content/EN/ TXT/HTML/?uri=CELEX:32019R0881\&from=EN.

[154] J. M. Porup. 2016. Underwriters Labs refuses to share new IoT cybersecurity standard. Retrieved from https://arstechnica.com/information-technology/2016/04/underwriters-labs-refuses-to-share-new-iotcybersecurity-standard/.

[155] Yanzhen Qu and Philip Chan. 2016. Assessing vulnerabilities in Bluetooth low energy (BLE) wireless network based IoT systems. In Proceedings of the IEEE 2nd International Conference on Big Data Security on Cloud (BigDataSecurity), IEEE International Conference on High Performance and Smart Computing (HPSC), and IEEE International Conference on Intelligent Data and Security (IDS). IEEE, New York, NY, 42-48. DOI : https://doi.org/10.1109/BigDataSecurityHPSC-IDS.2016.63

[156] Petar Radanliev, David C. De Roure, Jason R. C. Nurse, Rafael Mantilla Montalvo, and Peter Burnap. 2019. Standardisation of cyber risk impact assessment for the Internet of Things (IoT). (2019), 50. Retrieved from https: //www.preprints.org/manuscript/201903.0109/v2.

[157] RASEN project. 2015. D3.2.3. Techniques for Compositional Test-Based Security Risk Assessment v.3. Retrieved from http://www.rasenproject.eu/downloads/985/.

[158] Vinay Sachidananda, Shachar Siboni, Asaf Shabtai, Jinghui Toh, Suhas Bhairav, and Yuval Elovici. 2017. Let the cat out of the bag: A holistic approach towards security analysis of the Internet of Things. In Proceedings of the 3rd ACM International Workshop on IoT Privacy, Trust, and Security (IoTPTS'17). ACM Press, 3-10. DOI : https://doi.org/ $10.1145 / 3055245.3055251$

[159] Hunor Sandor and Gheorghe Sebestyen-Pal. 2017. Optimal security design in the Internet of Things. In Proceedings of the 5th International Symposium on Digital Forensic and Security (ISDFS'17). IEEE, 1-6. DOI: https://doi.org/10. 1109/ISDFS.2017.7916496 
[160] Martin Schneider, Jurgen Grossmann, Ina Schieferdecker, and Andrej Pietschker. 2013. Online model-based behavioral fuzzing. In Proceedings of the IEEE 6th International Conference on Software Testing, Verification and Validation Workshops. IEEE, 469-475. DOI : https://doi.org/10.1109/ICSTW.2013.61

[161] Robert C. Seacord. 2014. CERT C Coding Standard, Second Edition: 98 Rules for Developing Safe, Reliable, and Secure Systems. Addison-Wesley Professional, Upper Saddle River, NJ.

[162] SEI CERT. 2016. Coding Standards. Retrieved from https://wiki.sei.cmu.edu/confluence/display/seccode/SEI+CERT+ Coding+Standards.

[163] SEI CERT. [n.d.]. SEI CERT Perl Coding Standard. Retrieved from https://wiki.sei.cmu.edu/confluence/display/perl.

[164] Alireza Shameli-Sendi, Rouzbeh Aghababaei-Barzegar, and Mohamed Cheriet. 2016. Taxonomy of information security risk assessment (ISRA). Comput. Secur. 57 (Mar. 2016), 14-30. DOI : https://doi.org/10.1016/j.cose.2015.11.001

[165] Z. Shelby, K. Hartke, and C. Bormann. 2014. The Constrained Application Protocol (CoAP) (RFC7252). Retrieved from https://tools.ietf.org/html/rfc7252.

[166] V. L. Shivraj, M. A. Rajan, and P. Balamuralidhar. 2017. A graph theory based generic risk assessment framework for internet of things (IoT). In Proceedings of the IEEE International Conference on Advanced Networks and Telecommunications Systems (ANTS'17). IEEE, 1-6. DOI : https://doi.org/10.1109/ANTS.2017.8384121

[167] Sabrina Sicari, Alessandra Rizzardi, Daniele Miorandi, and Alberto Coen-Porisini. 2018. A risk assessment methodology for the Internet of Things. Comput. Commun. 129 (Sept. 2018), 67-79. DOI : https://doi.org/10.1016/j.comcom. 2018.07.024

[168] Saijda Sorsa. 2018. Protocol Fuzz Testing as a Part of Secure Software Development Life Cycle. Ph.D. Dissertation. Tampere University of Technology. Retrieved from https://dspace.cc.tut.fi/dpub/bitstream/handle/123456789/25667/ Sorsa.pdf? sequence $=3$.

[169] International Organization for Standardization. 2018. ISO/IEC 31000 - Risk Management. IEC. Retrieved from https://www.iso.org/iso-31000-risk-management.html.

[170] Bernard Stepien and Liam Peyton. 2014. Innovation and evolution in integrated web application testing with TTCN-3. Int. F. Softw. Tools Technol. Transf. 16, 3 (June 2014), 269-283. DOI: https://doi.org/10.1007/s10009-0130278- $\mathrm{x}$

[171] Michael Sutton, Adam Greene, and Pedram Aminir. 2007. Fuzzing-Brute force vulnerability discovery. AddisonWesley Professional, 1-51.

[172] Farid Molazem Tabrizi and Karthik Pattabiraman. 2016. Formal security analysis of smart embedded systems. In Proceedings of the 32nd Annual Conference on Computer Security Applications (ACSAC'16). ACM Press, 1-15. DOI : https://doi.org/10.1145/2991079.2991085

[173] Martin Tappler, Bernhard K. Aichernig, and Roderick Bloem. 2017. Model-based testing IoT communication via active automata learning. In Proceedings of the IEEE International Conference on Software Testing, Verification and Validation (ICST'17). 276-287. DOI : https://doi.org/10.1109/ICST.2017.32

[174] Emmeline Taylor and Katina Michael. 2016. Smart toys that are the stuff of nightmares. IEEE Technol. Soc. Mag. 35, 1 (Mar. 2016), 8-10. DOI : https://doi.org/10.1109/MTS.2016.2527078

[175] Ralf Tonjes, Eike Steffen Reetz, Klaus Moessner, and Payam Barnaghi. 2012. A test-driven approach for life cycle management of Internet of Things enabled services. In Proceedings of the Future Network and Mobile Summit. Retrieved from http://info.ee.surrey.ac.uk/Personal/P.Barnaghi/doc/IoTest-Paper.pdf.

[176] Petar Tsankov, Mohammad Torabi Dashti, and David Basin. 2012. SECFUZZ-Fuzz-testing security protocols. In Proceedings of the 7th International Workshop on Automation of Software Test (AST'12). IEEE, 1-7. DOI: https://doi. org/10.1109/IWAST.2012.6228985

[177] Underwriters Laboratories. 2017. UL 2900 Standards Process. Retrieved from https://industries.ul.com/ cybersecurity/ul-2900-standards-process.

[178] Underwriters Laboratories (UL). 2017. Software Cybersecurity for Network-Connectable Products, Part 2-1: Particular Requirements for Network Connectable Components of Healthcare and Wellness Systems. Retrieved from https://standardscatalog.ul.com/standards/en/standard_2900-2-1.

[179] Margus Valja, Matus Korman, and Robert Lagerstrom. 2017. A study on software vulnerabilities and weaknesses of embedded systems in power networks. In Proceedings of the 2nd Workshop on Cyber-Physical Security and Resilience in Smart Grids (CPSR-SG'17). ACM Press, 47-52. DOI : https://doi.org/10.1145/3055386.3055397

[180] VERACODE. 2006. VerAfied Methodology. Retrieved from https:/help.veracode.com/reader/ kJC1iOtXp8N rCtV8P9jhw/UQa oUCwYhluVREDo4480g.

[181] Alexandre Vernotte. 2013. Research questions for model-based vulnerability testing of web applications. In Proceedings of the IEEE 6th International Conference on Software Testing, Verification and Validation. IEEE, 505-506. DOI : https://doi.org/10.1109/ICST.2013.82

[182] Vasaka Visoottiviseth, Phuripat Akarasiriwong, Siravitch Chaiyasart, and Siravit Chotivatunyu. 2017. PENTOSPenetration testing tool for Internet of Thing devices. In Proceedings of the IEEE Region 10 Conference (TENCON'17) 2279-2284. DOI : https://doi.org/10.1109/TENCON.2017.8228241 
[183] Jeffrey Voas and Phillip A. Laplante. 2018. IoT’s certification quagmire. (Apr. 2018). DOI : https://doi.org/10.1109/MC 2018.2141036

[184] Dong Wang, Xiaosong Zhang, Ting Chen, and Jingwei Li. 2019. Discovering Vulnerabilities in COTS IoT Devices through Blackbox Fuzzing Web Management Interface. DOI : https://doi.org/10.1155/2019/5076324

[185] Huan Wang, Zhanfang Chen, Jianping Zhao, Xiaoqiang Di, and Dan Liu. 2018. A vulnerability assessment method in industrial Internet of Things based on attack graph and maximum flow. IEEE Access 6 (2018), 8599-8609. DOI : https://doi.org/10.1109/ACCESS.2018.2805690

[186] Zhongru Wang, Yuntao Zhang, Zhihong Tian, Qiang Ruan, Tong Liu, Haichen Wang, Zhehui Liu, Jiayi Lin, Binxing Fang, and Wei Shi. 2019. Automated vulnerability discovery and exploitation in the Internet of Things. Sensors 19, 15 (July 2019). DOI : https://doi.org/10.3390/s19153362

[187] Weibull. 2004. Basic concepts of FMEA and FMECA. ([n.d.]). Retrieved from http://www.weibull.com/hotwire/ issue 46/relbasics46.htm.

[188] Chanoksuda Wongvises, Assadarat Khurat, Doudou Fall, and Shigeru Kashihara. 2017. Fault tree analysis-based risk quantification of smart homes. In Proceedings of the 2nd International Conference on Information Technology (INCIT'17). IEEE, 1-6. DOI : https://doi.org/10.1109/INCIT.2017.8257865

[189] Tianshui Wu and Gang Zhao. 2014. A novel risk assessment model for privacy security in Internet of Things. Wuhan Univ. f. Nat. Sci. 19, 5 (Oct. 2014), 398-404. DOI : https://doi.org/10.1007/s11859-014-1031-3

[190] Dianxiang Xu, Manghui Tu, Michael Sanford, Lijo Thomas, Daniel Woodraska, and Weifeng Xu. 2012. Automated security test generation with formal threat models. IEEE Trans. Depend. Sec. Comput. 9, 4 (July 2012), 526-540. DOI : https://doi.org/10.1109/TDSC.2012.24

[191] Guangquan Xu, Yan Cao, Yuanyuan Ren, Xiaohong Li, and Zhiyong Feng. 2017. Network security situation awareness based on semantic ontology and user-defined rules for Internet of Things. IEEE Access 5 (2017), 21046-21056 DOI : https://doi.org/10.1109/ACCESS.2017.2734681

[192] Haiyun Xu, Jeroen Heijmans, and Joost Visser. 2013. A practical model for rating software security. In Proceedings of the IEEE 7th International Conference on Software Security and Reliability. IEEE, 231-232. DOI : https://doi.org/10. 1109/SERE-C.2013.11

[193] S. Yoo and M. Harman. 2012. Regression testing minimization, selection and prioritization: A survey. Softw. Test. Verif. Reliab. 22, 2 (Mar. 2012), 67-120. DOI : https://doi.org/10.1002/stv.430

[194] Yaowen Zheng, Ali Davanian, Heng Yin, Chengyu Song, Hongsong Zhu, and Limin Sun. 2019. FIRM-AFLHigh-throughput greybox fuzzing of IoT firmware via augmented process emulation. 1099-1114. Retrieved from https://www.usenix.org/conference/usenixsecurity19/presentation/zheng.

[195] Changying Zhou and Stefano Ramacciotti. 2011. Common criteria: Its limitations and advice on improvement. ISSA fournal (2011). Retrieved from https://www.difesa.it/SMD_/Staff/Reparti/II/CeVa/Pubblicazioni/Estere/Documents/ CommonCriteria_ISSA\%20Journal_0411.pdf.

[196] Wei Zhou, Yan Jia, Yao Yao, Lipeng Zhu, Le Guan, Yuhang Mao, Peng Liu, and Yuqing Zhang. 2019. Discovering and understanding the security hazards in the interactions between IoT devices, mobile apps, and clouds on smart home platforms. 1133-1150. Retrieved from https://www.usenix.org/conference/usenixsecurity19/presentation/zhou.

Received July 2019; revised December 2019; accepted July 2020 